\title{
Gas Turbine Rotor System Fault Diagnosis Method based on Improved Deep Convolutional Generative Adversarial Networks
}

Shucong Liu ( $\square$ liushucong@bistu.edu.cn )

Beijing Information Science \& Technology University

Hongjun Wang

Beijing Information Science \& Technology University

Fengxia Han

Beijing Information Science \& Technology University

Xiang Zhang

Beijing Information Science \& Technology University

\section{Research Article}

Keywords: Gas turbine rotor, Fault diagnosis, Improved Deep Convolutional Generative Adversarial Network, gradient penalty

Posted Date: November 30th, 2021

DOI: https://doi.org/10.21203/rs.3.rs-1086426/v1

License: (c) (1) This work is licensed under a Creative Commons Attribution 4.0 International License. Read Full License 


\title{
Gas Turbine Rotor System Fault Diagnosis Method based on Improved Deep Convolutional Generative Adversarial Networks
}

\author{
ShucongLiu ${ }^{1,2,3}$ Hongjun Wang ${ }^{1,3^{*}}$ Fengxia Han ${ }^{1,3}$,Xiang Zhang ${ }^{1,3}$ \\ (1 School of Mechanical and Electrical Engineering, Beijing Information Science and Technology University, \\ Beijing 100192, China \\ 2 College of Mechanical Engineering and Applied Electronics Technology, Beijing University of Technology, \\ Beijing 100124, China
}

3 Key Laboratory of Modern Measurement and Control Technology of Ministry of Education, Beijing Information Science and Technology University, Beijing 100192, China)

\begin{abstract}
:
In gas turbine rotor system fault diagnosis intelligent method based on data-driven is an important means to monitor the health status of gas turbine, it is necessary to obtain sufficient effective fault data to train the intelligent diagnosis model. In the actual operation of gas turbine, the collected gas turbine fault data is limited, and the small and imbalanced fault samples seriously affect the accuracy of fault diagnosis method. Aiming at the imbalance of gas turbine fault data, an Improved Deep Convolutional Generative Adversarial Network (Improved DCGAN) suitable for gas turbine signal is proposed, an structural optimization on generator of Deep Convolutional Generative Adversarial Network (DCGAN) and gradient penalty improvement on the loss function are introduced to generate effective fault data and improve the classification accuracy. The experiment results of gas turbine test bench demonstrated that the proposed method generated effective fault samples as a supplementary set of fault samples to balance the dataset, effectively improved the fault classification and diagnosis performance of gas turbine rotor in the case of small samples, The proposed method can be used as a solution to the problems of small unbalanced fault samples, and provides an effective method for gas turbine fault diagnosis.
\end{abstract}

\section{Keywords: Gas turbine rotor; Fault diagnosis; Improved Deep Convolutional Generative Adversarial Network; gradient penalty}

\section{Introduction}

As an internal combustion type rotating power machinery, gas turbine is the core component of power equipment in industrial fields, and it has developed rapidly and has been widely used in more fields such as aerospace, industrial power generation, navigation and land transportation. The reliability and safety of gas turbines are getting more and more attention. Due to various unavoidable factors, the gas turbine has various failures, and the predetermined function is reduced or even completely lost. As a result, serious or even catastrophic accidents are caused, and huge economic losses are caused to enterprises and even industries [1].

Common gas turbine faults are divided into two categories: the first is related to aerodynamics or performance-based, such as thermal distortion, compressor erosion and corrosion, compressor fouling, turbine fouling, turbine erosion and corrosion, blade rubbing, etc. Gas path analysis (GPA) is an effective technical means for forecasting these faults [2]. At present, machine learning based on data-driven have been used for gas path diagnosis based on fault sample set, such as Artificial Neural Network (ANN) [3], Support Vector Machine(SVM)[4],Bayesian network [5], fuzzy logic [6].The second is related to mechanical properties, such as shaft misalignment, rotor dynamic imbalance, bearing defects and oil film instability [7]. For such faults, there are many technical means, such as oil chip analysis, vibration analysis, acoustic analysis, thermal aging, load analysis, metal temperature, and stress analysis and so on.

Vibration monitoring [8] is one of the commonly used methods for gas turbine condition monitoring and fault diagnosis. Effective condition monitoring, fault diagnosis, and maintenance are effective means to ensure the safe, reliable and stable operation of gas turbines. Through vibration sensors arranged in the casing and other parts of the gas turbine, the vibration signals of the gas turbine are obtained and analyzed with certain signal processing technology to extract the vibration characteristics for locating the fault and finding out the cause of the fault, thereby providing a basis for the diagnosis, evaluation and decision-making of the gas turbine. Vibration condition monitoring can reduce downtime for maintenance, reduce maintenance costs, reduce safety accidents, and ensure 
the normal operation of equipment to improve its reliability [9].

The rotor system is the most important component in gas turbine. Due to the complex structure of the gas turbine rotor system, and strict assembly process, it plays a vital role in the healthy operation of the gas turbine. The rotor system runs under harsh working environment with high temperature, high pressure, and high speed, and it is very easy to collide with the internal structure. Once a failure occurs, the rotor will deviate from the normal working state, and it will cause huge damage to the gas turbine in severe cases[10].

In gas turbine rotor system, rotor imbalance fault, misalignment fault, rotor rub impact fault, rotor thermal bending and other faults emerges. Through vibration monitoring and analyzing the vibration signal of rotor, fault diagnosis and condition evaluation can be carried out to supply suggestions for maintenance personnel and is of great significance to ensure the safe and reliable operation of gas turbine rotor system.

The development of gas turbine condition monitoring and fault diagnosis methods can be divided into three stages: methods based on qualitative experience knowledge, methods based on model analysis, and methods based on data-driven. The method based on qualitative empirical knowledge relies on the manual judgment of field experts and do simple processing on the monitoring data. In the method based on model analysis, sensors are used to monitor each component of the engine, signal processing is performed, and engine mathematical models, statistics analytical model or artificial intelligence pattern recognition model based on observation data are established. In the data-driven method, mathematical models, statistical analysis models and machine learning models are integrated, and expert experiences, knowledge about engines and information such as engine monitoring data are merged. Among them, the intelligent diagnosis method is a new method using many new methods and means, including fuzzy logic, expert system and artificial neural network [11]. With the continuous application development and improvement of these methods, the condition monitoring of mechanical equipment will gradually be systematic and intelligent. At present, data-driven diagnostic methods mainly include: statistical analysis methods, signal processing methods and artificial intelligence-based methods [12]. Among them, statistical methods are limited to statistical analysis theories, and their scope of application is limited. Signal processing technology is an important means of mechanical vibration fault analysis, represented by Fourier transform [13], wavelet packet analysis [14], Hilbert-Huang transform [15] and other methods. The signal processing method is based on the signal analysis technology to extract the time-frequency domain characteristic parameters and characterize system state, and there will still be data loss in this process [16].

The fault diagnosis method based on artificial intelligence technology does not require the establishment of professional related mathematical models, and the application of advanced classification and regression algorithms to historical data or running logs can realize fault diagnosis [17]. In many fault diagnosis based on artificial intelligence technology, typical representatives are methods based on artificial neural networks [18], methods based on support vector machines [19], and methods based on fuzzy logic [20]. At the same time, the classic classification methods of machine learning also include decision trees, random forests, and extreme decision trees and so on. With the breakthrough in the performance of artificial intelligence algorithms, many advanced data processing methods, image recognition tools and data analysis algorithms have gradually been applied in the prototype design, method optimization and fault diagnosis of gas turbines [21-24], and many results have been achieved.

The progress of deep learning also has some applications in gas turbine fault diagnosis[25-27].However, most of the intelligent diagnosis methods based on deep learning require sufficient data samples, and the problem of fault data scarcity in gas turbines actual engineering is a big challenge for gas turbines application. At present, due to the complex structure of the large gas turbine rotor system and the limited test conditions, there are only some fault data under each working condition of practical running, and some faults have never occurred. For the fault types not involved in the sample set, it is difficult to obtain accurate diagnosis results by using deep learning methods. In addition, individual differences such as equipment manufacturing and installation errors and changes in operating conditions make the application scenarios of equipment complex and changeable, the applicability of the method needs to be improved.

Therefore, how to solve the problems of small sample, insufficient labeled data, and low diagnosis accuracy has become the top priority of fault diagnosis and identification. Generative Adversarial Network (GAN) as artificial intelligence method provides a good technical means to solve this problem [28-29]. Its unique adversarial idea makes it stand out among many generator models, and has wide applications in sample generation, data enhancement and other fields. In the generative adversarial network, the existence of the generator makes the sample size and completeness no longer become the key factors that affect the accuracy of the entire network. Luo et al. [30] proposed a new generative adversarial learning model to deal with the fault diagnosis of rotating machinery. The generator can generate fault data with higher reliability to improve the fault diagnosis data set. Wang et al. [31] aimed at the problem of sample imbalance, obtained the fault characteristics of the signal through GAN, and generated new fault samples. Viola et al. [32] proposed a generative model based on a Generative Adversarial Networks to generate synthetic samples that conform to the original data sample distribution, and 
extended training samples to solve the overfitting problem of the classification model due to insufficient training samples. Li et al. [33] used the Enhanced Generative Adversarial Network to compare the probability distribution between the generated signals and the real signals, and generated the fault signals for rotating machinery fault diagnosis.

DCGAN is an improvement model of GAN [34]. It mainly improves the network structure of GAN. It combines the automatic feature extraction layer of the sample with the GAN network, which greatly improves the stability of GAN training and the quality of generated data [35-36]. However, the signal generator of the traditional GAN or DCGAN generates the sample signals based on random data. The random noises generated each time during the operation is a special code that only a group of generators understand in a sense, and it determines how the generated result should be represented. The result generated by using random data as the encoding source has little relation with the original signals, making the generator prone to produce meaningless output, resulting in unstable network training and learning, and low quality of the generated signals. Therefore, an improved method based on the original DCGAN to generate sample data is proposed and applied to gas turbine rotor system fault diagnosis effectively. Firstly, the proposed method combines the data generation ability of DCGAN and the feature extraction ability of CNN to construct a new optimized signal generator, which further improves the quality of generative fault samples for fault diagnosis. Then generative samples are combined with original samples to form feasibility samples and to supplement the gas turbine rotor faults dataset, the fault samples are adopted to build up fault diagnosis model. Finally, test on gas turbine test bench experiment are carried out for fault classification and verify the proposed method. The main contributions can be summarized as follows:

(1)The improved DCGAN is proposed and the generator is optimized by adding one-dimensional deep convolutional neural network layer with a wide first-layer kernel(WDCNN)to the deconvolution layer of generator, to build generator containing the gas turbine vibration data features and generate samples with higher quality and higher similarity with original samples.

(2)The loss function of generator and discriminator are optimized with gradient penalty is adopted to solve the problem of gradient disappearance and improve the applicability of the method. The optimized penalty factor can be adjusted according to the actual situation, which improves the adaptability of the method.

(3)The generated effective fault samples are added to the fault dataset, and the proposed method is validated on the gas turbine rotor system fault data to solve the problem of insufficient gas turbine rotor system faults samples and provide new ideas for gas turbine rotor system fault diagnosis.

The remainder of this paper proceeds as follows. Section1 is the introduction, Section2 presents theoretical method, Section3 is the proposed fault diagnosis method, Section 4 is the experiment and results, Section5 conclude the paper.

\section{Theoretical method introduction}

\subsection{Deep Convolutional Generative Adversarial Network（DCGAN）}

Generative Adversarial Network is a machine learning architecture proposed by Ian Goodflow in 2014, and it is one of the most promising methods of unsupervised learning in recent years. Due to its outstanding data generative capabilities, generative adversarial networks have been fully applied in many fields, especially in image processing, text generation, audio processing, data enhancement and sample prediction. The main idea of GAN is derived from game theory, in which a discriminative model and a generative model are setup to compete with each other to improve the learning effect and obtain high-dimensional and complex data distribution of real samples. Its network structure is shown in Fig.1:

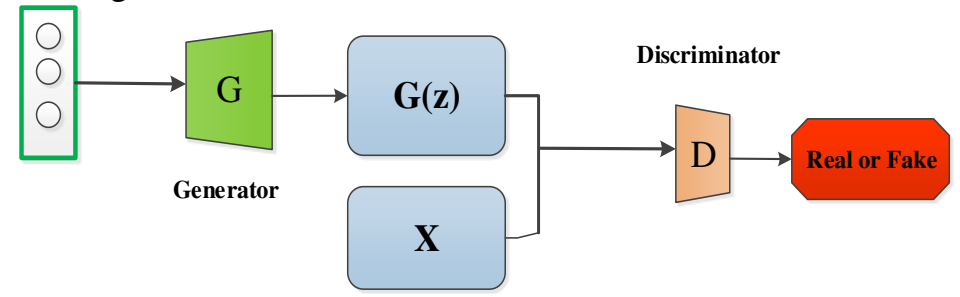

Fig.1 GAN network structure diagram

The main structure of GAN includes a generator and a discriminator[37-38]. The generator is used to generate real samples, and the discriminator is used to determine real samples and fake samples. The generator $G$ inputs a random noise vector $z$ (usually uniform or normal distribution)and maps it to a new multi-dimensional data space to achieve generated fake sample $G(z)$, the discriminator $D$ is used to perform classification and to calculate the probability that testing sample is a real sample. In the training, the generator $G$ try the best to learn the data distribution of the real sample to generate fake sample, and the discriminator $D$ try to distinguish the generated 
fake sample from real sample. The objective function of the generated adversarial network isas follows:

$$
\min _{G} \max _{D} V(G, D)=E_{x \sim P_{\text {data }}(x)}[\log D(x)]+E_{z \sim P_{z}(z)}[\log (1-D(G(z)))]
$$

During the training process of GAN network, the objective function of $D(x)$ is

$$
\max \underset{D}{V}(D, G)=E_{x \sim P_{\text {data }}(x)}[\log (D(x))]+E_{z \sim P_{z}(z)}[\log (1-D(G(z)))]
$$

The parameters are optimized by Eq.(2) to make $D(x)$ to obtain the best.The objective function of $G$ is

$$
\min V_{G}(D, G)=E_{z \sim P_{z}(z)}[\log (1-D(G(z)))]
$$

Among them, $x$ represents the data sample, $P_{z}(z)$ represents the data distribution of the input noise, $G(z)$ represents sample generated by the input noise, $D(x)$ represents the probability of sample $x$ determined by the discriminator is the real sample instead of the generated sample. $E_{x \sim P_{\text {data }}(x)}[\log D(x)]$ represents the probability of $D(x)$ obtaining real data; $E_{x \sim P_{z}(z)}[\log (1-D(G(z)))]$ represents the probability of $D(x)$ discriminating real data.

During the training process, alternate iterations are performed. Firstly the network $G$ is fixed, the discriminator network $D$ is trained so that the labels of the training samples are classified correctly with the greatest probability, that is maximize $\log D(x)$ and $\log (1-D(G(z)))$. Then the network $D$ is fixed, the network $G$ is trained to minimize $\log (1-D(G(z)))$. The training of the generator network makes $D(G(z))$ be close to 1 , so that the generator loss will be the smallest. The goal of training discriminator network is to distinguish the real data and the generated data, that is, the discriminator output of the real data is expected to be close to 1 , and the output of the generated data $D(G(z))$ is close to 0 . Finally the training reach to balance, and $G$ could estimate the distribution of sample data.

The focus of GAN is to find the Nash equilibrium point, and the goal of the gradient descent method in the neural network is to find the smallest loss function, so in model training there may be situations where the model cannot converge or the convergence is unstable. Moreover, there may be a pattern collapse phenomenon in GAN, that is, the generated data sample pattern is single and it does not cover all categories of real data. Therefore, how to ensure stable training of GAN has always been a hot and difficult topic[39-40].

With the continuous intensive research in the field of image and sample generation by generative adversarial network, many excellent image and sample generation algorithms have emerged. Radford et al. [41] proposed a Deep Convolutional Generative Adversarial Network. By improving the neural network structure in the GAN model, deconvolution layer innovatively replaced the fully connected layer of the generator in the basic GAN network, thus achieving excellent performance in image generation tasks. As shown in Fig.2, the powerful feature extraction capability of CNN in DCGAN is adopted to improve the learning effect of the generative network. By use of Batch Normalization (BN) in the layer, the generator can learn stably, so that the model can learn better data samples distribution, more stably generate high-quality samples.

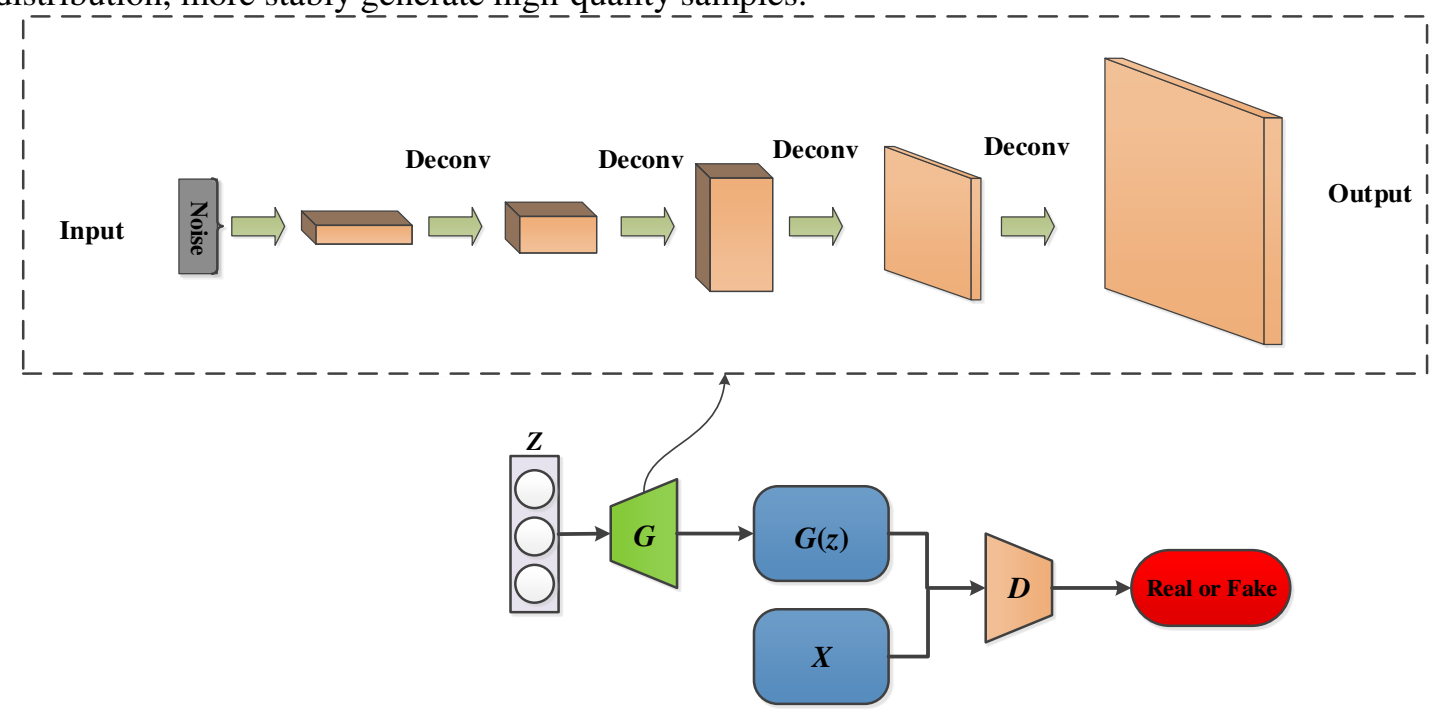

Fig.2 Structure of DCGAN 
DCGAN improves the GAN structure, and greatly improves the stability of GAN training and the quality of generated data [42-43]. Its main improvements are as follows: convolutional neural network is used instead of the original GAN generator and discriminator network structure, in generator network micro-step convolution is used instead of pooling layer and fully connected layer, in discriminator network step-size convolution is used instead of pooling layers and fully connected layers. Batch normalization unified operations are added to both the generator network and the discriminator network, and in order to prevent the oscillation and instability of CNN, batch normalization unified operation is not added to the output layer of the generator network and the input layer of the discriminator network. The tanh activation function is used in the output layer of the generator network, the ReLU activation function is used in all rest layers. In the discriminator network the LeakyReLU activation function is used instead of the ReLU activation function to solve the vanishing gradient problem when the discriminator network returns to the generator network. DCGAN greatly improves the stability of GAN training and the quality of generated results.

\subsection{Convolutional neural network (CNN)}

Convolutional Neural Network is a typical deep neural network. It reduces the complexity of the network model through the weight sharing method and improves the efficiency of model training. The advantage of this network is that the network model can be trained with input images or signals, eliminating the need for feature extraction. Convolutional Neural Network consists of input layer, convolution layer, pooling layer, fully connected layer and output layer. The input layer realizes the input of the original dataset, the feature is obtained through convolution calculation of the convolution layer, and the pooling layer performs down-sampling operation on the feature image to reduce the data dimension. The number of convolutional layers and down-sampling layers is often determined according to actual conditions. The fully connected layer, just as the neural network, mainly implements data mapping[44-45].

The idea of convolutional layer is to use convolution kernel to extract local features, which is similar to an automatic feature extraction, which has been widely used in feature extraction, fault diagnosis and so on. For time series vibration signals, one dimension Deep Convolutional Neural Network with a Wide first-layer kernel (WDCNN) has strong processing capabilities, which can eliminate complex feature extraction and directly perform operations on the sequence. Its main feature is that it has the first layer of large convolution core and multi-layer small convolution kernel with the size as $3 \times 1$. It can provide faster processing speed and it is suitable for real-time fault diagnosis. In the proposed method, one-dimensional deep convolutional neural network with a wide first-layer kernel is used for vibration signals feature extraction, and it is used as the coding part of the new generator.

The detailed settings of the proposed network structure is shown in Table 1. Vibration signals are used in the input layer. Different kernel sizes, number and stride have been selected to perform the one-dimension convolution operation with appropriate number. Such as Conv 1, the kernel size, number and stride are 32, 20 and 8, respectively. The BN and ReLU activation function are adopted after the Conv layer, and the pooling layer are used to perform the pooling operation. The dropout operation is used to after FC1 and FC2, and 10 different classifications can be recognized.

Table 1. Detailed settings of the WDCNN structure

\begin{tabular}{ccccc}
\hline Layer Type & Activation & Kernel Number & Kernel Size $\times$ Stride & Output Size \\
\hline Input & & & & $(1024,1)$ \\
Conv & ReLU & 20 & $32 \times 8$ & $125 \times 1 \times 20$ \\
BN & & & & $125 \times 1 \times 20$ \\
Pool & & 20 & $2 \times 2$ & $62 \times 1 \times 20$ \\
Conv & ReLU & 20 & $3 \times 1$ & $62 \times 1 \times 20$ \\
BN & & & & $62 \times 1 \times 20$ \\
Pool & & 20 & $3 \times 2$ & $31 \times 1 \times 20$ \\
Conv & ReLU & 20 & & $31 \times 1 \times 20$ \\
BN & & & $2 \times 2$ & $31 \times 1 \times 20$ \\
Pool & & 20 & $3 \times 1$ & $15 \times 1 \times 20$ \\
Conv & ReLU & 20 & & $15 \times 1 \times 20$ \\
BN & & & $2 \times 2$ & $7 \times 1 \times 20$ \\
Pool & & 20 & & \\
\hline
\end{tabular}

\subsection{Maximum Mean Discrepancy (MMD)}

The MMD algorithm is a loss function widely used in transfer learning. It is mainly used to measure the distance of the distribution of two different but related sample data and observe the distribution differenceof the two types sample data[46]. In the proposed DCGAN method, MMD is used to measure the similarity between generated samples and original samples. The smaller the MMD, the better the similarity between the generated sample and the original sample, the higher quality of generated samples. 
MMD mainly used high-dimensional mapping functions to find the expected difference in RHKS of the two samples to determine the similarity[47]. Assume the samples obey the $p$ distribution $\mathrm{D}_{s}=\left\{x_{1}, x_{2}, \cdots, x_{n}\right\}$ and the samples obey the $q$ distribution $\mathrm{D}_{m}=\left\{y_{1}, y_{2}, \cdots, y_{m}\right\}$, function $\phi: \chi \rightarrow H$ maps samples to high-dimensional RKHS, in which $\chi$ is the feature space of the sample. The distribution of different samples in RKHS is effectively matched by means of kernel mean embedding. For the unbiased estimation of the kernel average embedding is obtained by calculating the expected value $E_{p}\left[\phi\left(x_{i}\right)\right]$ and $E_{q}\left[\phi\left(\mathrm{y}_{i}\right)\right]$ of the sample mapped to the RKHS. In which $\phi\left(x_{i}\right)$ is the representation of sample $x_{i}$ mapped to RKHS through high-dimensional functions.MMD is expressed as follow:

$$
\operatorname{MMD}^{2}(p, q) \square \sup _{\phi \in H}\left\|E_{p}\left[\phi\left(\mathrm{D}_{n}\right)\right]-E_{q}\left[\phi\left(\mathrm{D}_{m}\right)\right]\right\|_{H}^{2}=\left\|\frac{1}{n} \sum_{i=1}^{n} \phi\left(x_{i}\right)-\frac{1}{m} \sum_{j=1}^{n} \phi\left(y_{j}\right)\right\|_{H}^{2}
$$

RKHS is a complete high-dimensional inner product space. Dot product operation of $\phi\left(x_{i}\right)$ and $\phi\left(y_{j}\right)$ can be calculated with kernel function, generally Radial Basic Function (RBF) is chose to represent the infinite dimensions Gaussian kernel:

$$
\left\langle\phi\left(x_{i}\right), \phi\left(y_{i}\right)\right\rangle_{H_{k}}=k\left(x_{i}, y_{j}\right)=\exp \left(-\left\|x_{i}-y_{i}\right\|^{2} / \sigma\right)
$$

Among them: $\sigma$ represents the bandwidth of the Gaussian kernel. Rewrite Eq. (5) by Eq. (4), namely:

$$
\operatorname{MMD}^{2}(p, q) \Gamma\left\|\frac{1}{n} \sum_{i=1}^{n} \phi\left(x_{i}\right)-\frac{1}{m} \sum_{j=1}^{n} \phi\left(y_{j}\right)\right\|_{H_{k}}^{2}=\frac{1}{n(n-1)} k\left(x_{i}, y_{j}\right)+\frac{1}{m(m-1)} \sum_{i \neq j}^{m} k\left(y_{i}, y_{j}\right)-\frac{2}{n m} \sum_{i, j=1}^{n, m} k\left(x_{i}, y_{j}\right)
$$

It can be seen from Eq. (6), when $p=q, M M D^{2}(p, q)=0$; when $p \neq q$,it needs to find the mapping function $k(\bullet)$ to minimize the $\operatorname{MMD}^{2}(p, q)$, so that the probability distributions of the two samples in RKHS are more similar under the representation $\phi(\bullet)$ to reduce the distribution difference in RKHS. In the improved DCGAN method, new faults samples are generated by generative adversarial network, and MMD is used to measure the similarity between generated samples and real samples. The smaller the MMD, the better the similarity between the generated sample and the original sample.

\section{3 .Gas turbine rotor system fault diagnosis method based on improved DCGAN}

\subsection{The Improved DCGAN}

In traditional GAN generally random noises are used as signal sources to enter into the generator $G$, and new signals and samples are generated after processing by the network. In order to make the generated signal consistent with the real signal, a discriminator $D$ is trained and used to identify whether the signal is real data or fake data generated by the generator. The random noise generated each time during the operation is a special code that only a group of generators understand in a sense, and it determines how the generated result should be represented. The result generated by using random data as input has little relationship with the original signals, leading to low similarity between the signals generated by the generator and original sample signals and long training time of the network.

Each convolutional layer in a convolutional neural network consists of several convolutional units, and the parameters of each convolutional unit are optimized through the backpropagation algorithm. The purpose of the convolution operation is to extract different features of the input. The first convolution layer may only extract some low-level features such as edges, lines, and corners. The network with more layers can iteratively extract more complex features from the low-level features. Transpose Convolution Layer can in turn synthesize pictures or signals based on features, and has been used to generate adversarial networks such as DCGAN.

If the advantages of the convolutional layer and the transposed convolutional layer in CNN can be combined with each other, a new generator can be formed. Therefore, in order to obtain the generated samples with higher similarity to the original samples, an improved DCGAN method is proposed. Fig3 is shown as the structure of improved DCGAN. On the basis of DCGAN, the trained WDCNN network are introduced to the generator, the features of input fault signals are obtained through WDCNN network training, so the input contains fault features and the generated samples similar to the original samples are easier to be obtained through the generator.

The generator of the improved DCGAN network consists of three parts: an encoder network, the reshape network, and a decoder network. The encoder network extracts the feature representation of the input signals, and WDCNN are used as convolutional layers to construct the encoder. The reshape network consists of the project and 
reshapes Layer, which upscales the input using a fully connected operation and reshapes the output to the specified size. The decoder network consists of transposed convolutional layers, and takes sample the transformed features and outputs the final generated signals.

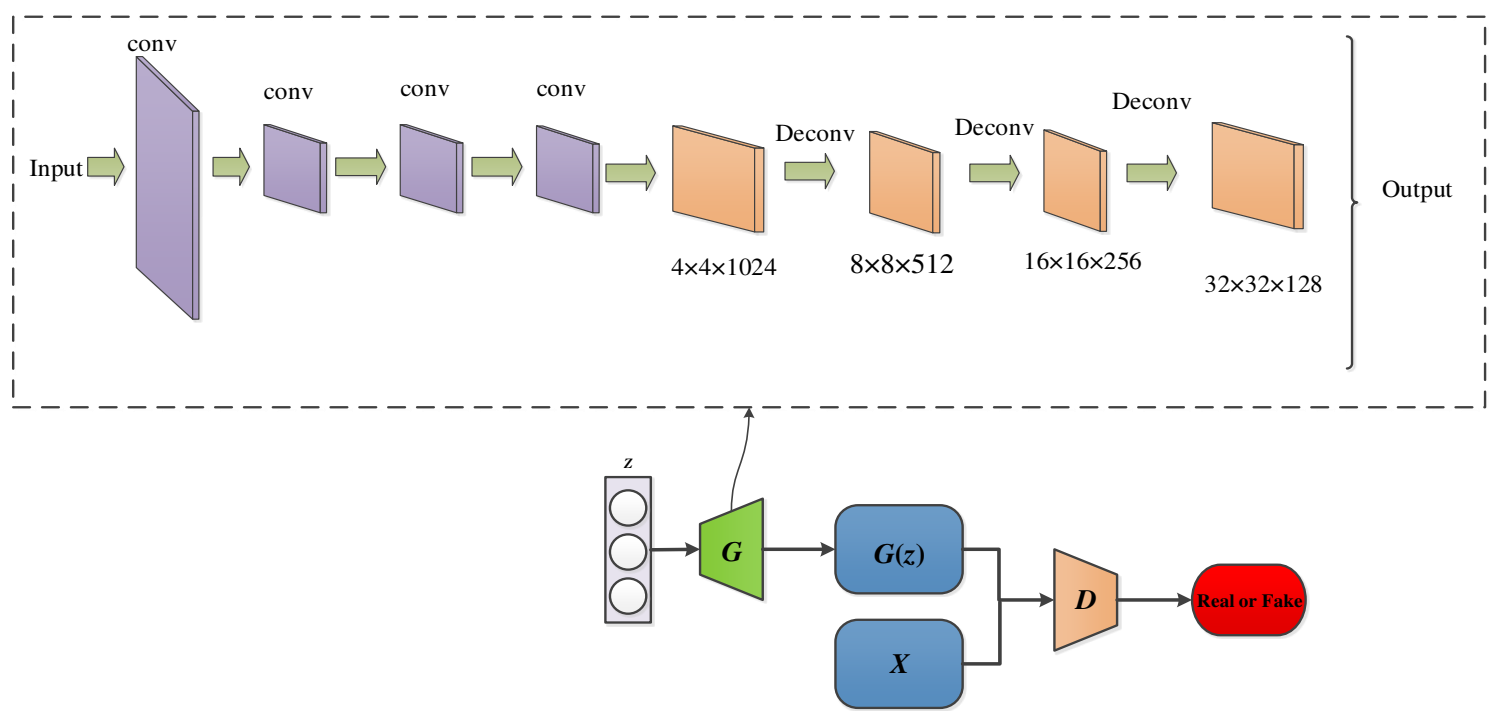

Fig.3 Structure of improved DCGAN

The discriminator examines patches of the input samples and determines the probability of the samples being real or fake. As can be observed, the discriminator has an input layer of $32 \times 1$. Also, three hidden layers are employed with LeakReLU as the activation function. Finally, the output layer has a dimension of $256 * 1$, which is fully connected with a Sigmoid activation function for the real and fake data classification. The kernel size for the CNN is $3 \times 1$ in all its layers with stride of 2 for all the hidden layers except by the output with striding of 1 .

Table1 Structure parameters and hyper-parameters of Improved DCGAN

\begin{tabular}{llllll}
\hline Model & $\begin{array}{l}\text { Network } \\
\text { layer }\end{array}$ & Kernel Size & Stride & $\begin{array}{l}\text { Activation } \\
\text { Function }\end{array}$ & $\begin{array}{c}\text { Kernel } \\
\text { Number }\end{array}$ \\
\hline Generator & Conv1 & 32 & 8 & ReLU & 20 \\
& Conv2 & 3 & 1 & ReLU & 20 \\
& Conv3 & 3 & 1 & ReLU & 40 \\
& Conv4 & 3 & 1 & ReLU & 40 \\
& Deconv1 & 3 & 1 & ReLU & 128 \\
& Deconv2 & 3 & 2 & ReLU & 64 \\
& Deconv3 & 3 & 1 & ReLU & 32 \\
& Deconv4 & 3 & 2 & Tanh & 1 \\
& Conv1 & 32 & 2 & LeakReLU & 32 \\
& Conv2 & 32 & 2 & LeakReLU & 64 \\
& Conv3 & 32 & 2 & LeakReLU & 128 \\
& Conv4 & 32 & 2 & LeakReLU & 256 \\
& Conv5 & 2 & 1 & & 1 \\
\hline
\end{tabular}

\subsection{Loss function optimization based on gradient penalty}

In the process of generative adversarial network training, the loss function is the key to the quality of network learning. If the loss function is incorrect, it will lead to training instability and even exploding gradient problem, and difficulty to train the correct model. The loss function in GAN is used still in DCGAN continuously. Based on DCGAN, an improved method with gradient penalty is adopted in the proposed method, in which original loss function in GAN is abandoned, additional gradient penalty is added to the loss function of discriminator, to solve vanishing gradient problem and exploding gradient problem in training. The sparse gradient penalty algorithm is used as the loss function in the process of model training, the negative gradient direction is used as the search 
direction, and the minimum value is solved along the gradient descent direction, so as to approach the minimum deviation in a recursive way. In the training process, after each/every forward propagation, the real values of loss value and output value will be obtained. The smaller the loss value, the better the model.

Firstly, the samples $x_{r}$ and $x_{g}$ are respectively obtained in the real sample space $P_{r}$ and the generated sample space $P_{g}$, then $\hat{x}$ is obtained by random interpolation between the real sample $x_{r}$ and the generated sample $x_{g}$, as shown in Eq. (7)and Eq. (8)

$$
\begin{aligned}
& \quad x_{r} \sim P_{r}, x_{g} \sim P_{g}, \varepsilon \sim U[0,1] \\
& \hat{x}=\varepsilon x_{r}+(1-\varepsilon) x_{g}
\end{aligned}
$$

$\varepsilon$ represents the random sampling of the samples. According to the joint space sampling $x$, the discriminator output $D(\hat{x})$ is made, and then the joint derivation is carried out.

$$
\operatorname{grad}=\sum_{i=1}^{N} \frac{\partial D(\hat{x})}{\partial \hat{x}}
$$

Where, ${ }^{i}$ represents the number of batch size samples. Eq.(9) is used for the square difference processing to the joint derivative ${ }^{\text {grad }}$. In order to adapt to the sample characteristics, finally the joint space sample loss function $\operatorname{loss}_{\text {eps }}$ is obtained after the square difference, the gradient penalty term is shown in Eq.(10):

$$
G P=E_{x \sim \lambda}\left[\left(\square \nabla_{x} D(x) \bigsqcup_{2}-1\right)^{2}\right]
$$

$\nabla_{x} D(x)$ represents the gradient of the discriminator output valuein the $\mathrm{X}$ direction; $E_{x \sim \lambda}$ represents the mathematical expectation when the input of the discriminator is random interpolation.

$$
\operatorname{losS}_{\text {eps }}=\lambda \times \frac{1}{N} \sum_{i=1}^{N_{\text {grad }}}\left(\operatorname{Re} \operatorname{lu}\left(\left(\sum_{i=1}^{N}\left(\operatorname{grad}^{2}\right)_{i}\right)^{\frac{1}{2}}-1\right)\right)
$$

$N_{\text {grad }}$ represents the rows number of the matrix $\mathrm{grad} ; \lambda$ represents the gradient penalty parameter.

The original loss function of the discriminator is shown in Eq. (12):

$$
D_{\text {original }}=E_{x \sim P_{r}}[D(x)]-E_{x \sim P_{g}}[D(x)]
$$

In which $E_{x \sim P_{g}}[D(x)]$ represents the mathematical expectation when the inputs of discriminator are generated samples; $E_{x \sim P_{r}}[D(x)]$ represents the mathematical expectation when the inputs of the discriminator are real samples.

In the discrimination of samples, the goal of the discriminator loss function is to reduce the gap between the predicted value of the model and the real value as much as possible. The difference between the loss function of discriminator discriminating real and fake samples are weighted and the final discriminator loss function is obtained.

$$
D_{\text {loss }}=\sum_{i=1}^{N} D\left(f_{\text {real }}\right)-\sum_{i=1}^{N} D\left(f_{\text {fake }}\right)+\text { loss } s_{\text {eps }}
$$

In the loss function of the generator, since the problem solved by the generator is different from that of the discriminator, the loss in the model is mainly caused by convolution processing. The loss caused by joint sampling samples is calculated according to the gradient descent method, and the loss function formula is as follows:

$$
\text { ploss }=\sum_{i=1}^{N}\left(\sum_{j=1}^{M}\left(\operatorname{out}\left(f_{\text {real }}, 1\right)-\text { out }\left(f_{\text {fake }}, 1\right)\right)_{j}\right)
$$

The final generator loss function $G_{\text {loss }}$ is as follows: 


$$
G_{\text {loss }}=\beta \times \frac{1}{N}\left(\sum_{i=1}^{N}\left(\text { ploss }-D\left(f_{\text {real }}\right)\right)_{i}\right)
$$

In which $\beta$ represents the gradient penalty parameter of the generator, which can be used to adjust the intensity of gradient penalty.

Compared with DCGAN, the new gradient penalty term will make the overall training of deep convolution generative adversarial network with gradient penalty more stable, and the convergence and training speed faster.

\subsection{The proposed gas turbine rotor system faults diagnosis method}

In traditional generative adversarial network random data is used as the encoding source to generate samples, in order to improve network training stability and the generated signals quality, the input introduce faults features in relation with the original signals. According to the improved DCGAN structure and optimized loss function, an improved method is proposed, which can be applied to gas turbine rotor faults diagnosis under different unknown conditions with small labeled samples, with the purpose of generating effective sample data and supply dataset for fault diagnosis. Fig. 4 is a flow chart of the proposed gas turbine rotor faults diagnosis method. The whole process is summarized as follows:

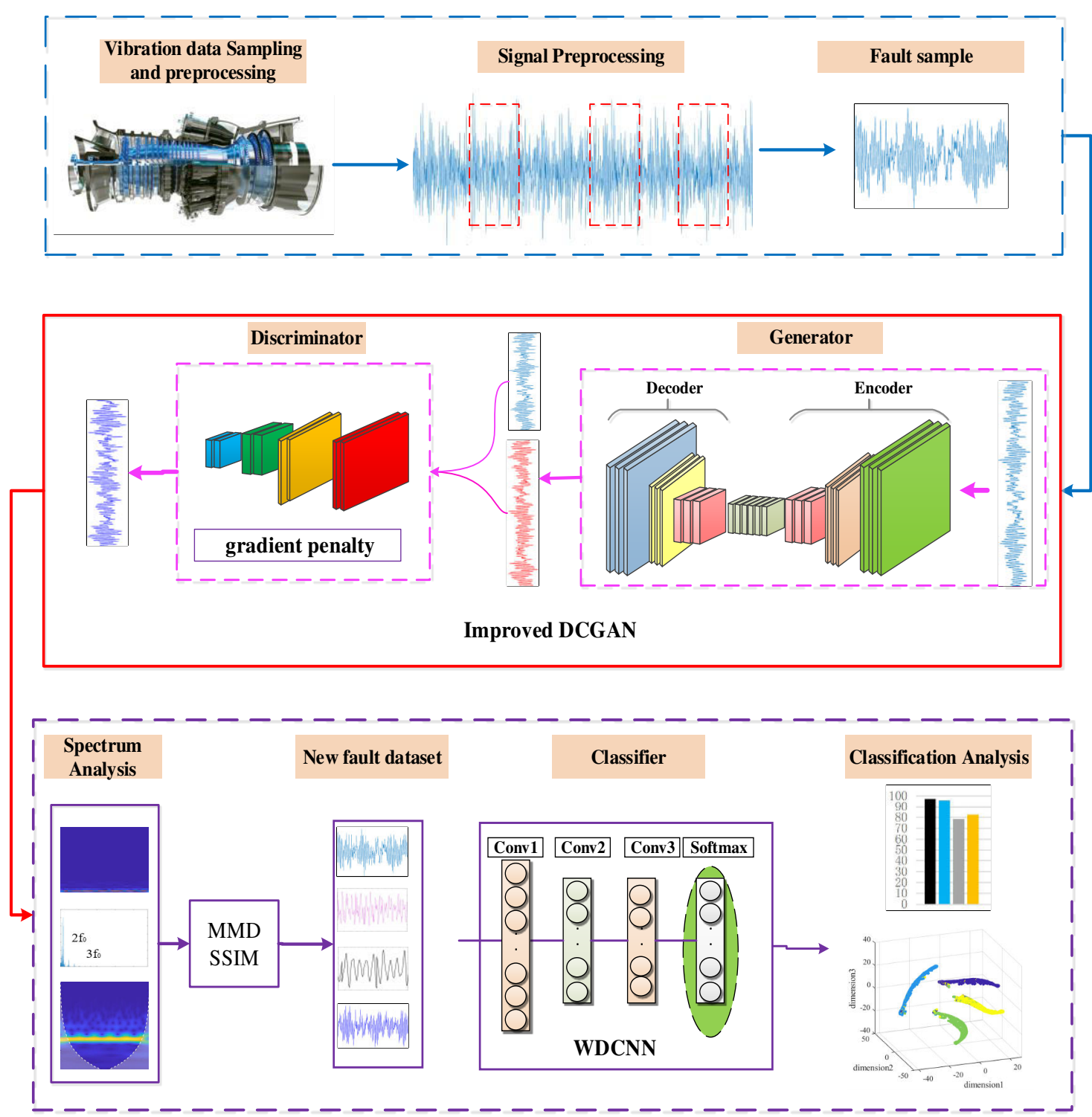

Fig. 4 Flow chart of the proposed gas turbine rotor fault diagnosis method

Firstly, accelerometers installed on different parts of the gas turbine are adopted to collect vibration data, and then the obtained signals are preprocessed by time domain and frequency domain analysis. Combining the analysis with expert diagnosis, labeled samples are obtained and the data are divided into training set and testing set;

Secondly, the faults samples are adopted as the input of proposed DCGAN network to generate faults samples. 
The one-dimensional CNN network suitable for vibration signal feature extraction is constructed, and the network and parameters are used for the generator of the improved DCGAN network, and a new generator for feature extraction of actual sample data is formed to continually generate new associated samples;

Thirdly: The sample maximum mean difference (MMD) method is used to determine the mean difference between the generated signal and the real signal, and combine the generated data that meets the requirements with the original small sample data to form a new data set;

Last: Faults classification and diagnosis are performed on the generated new sample set, and results comparisons and accuracy analysis are done to verify the method.

\section{Gas turbine test bench experiment and fault diagnosis \\ 4.1Experiment and data acquisition}

To further verify the performance of the proposed method, the gas turbine rotor system fault experiment from test bench is adopted. The rotor system of a gas turbine is shown in Fig.5, which is mainly composed of compressor, turbine, rotating shaft and so on. The low-pressure turbine is connected to the low-pressure compressor through the low-pressure rotating shaft, and the high-pressure turbine is connected to the high-pressure compressor through the high-pressure rotating shaft sleeved on the low-pressure rotating shaft. Due to the complex and high-temperature internal environment of gas turbine, the whole machine vibration measurement is often used in the engineering application. Through multiple experiments and analysis at different positions and angles, as shown in Fig.5, a speed sensor (sensor 1) is installed on the radial position at the front end of low-pressure compressor casing as the front measuring point, a speed sensor (sensor 2) is installed at the radial position of the casing between the high-pressure compressor and the combustion chamber as the rear measuring point, and the rotor speed sensor for measuring high and low pressure rotor speed is installed inside the gas engine.

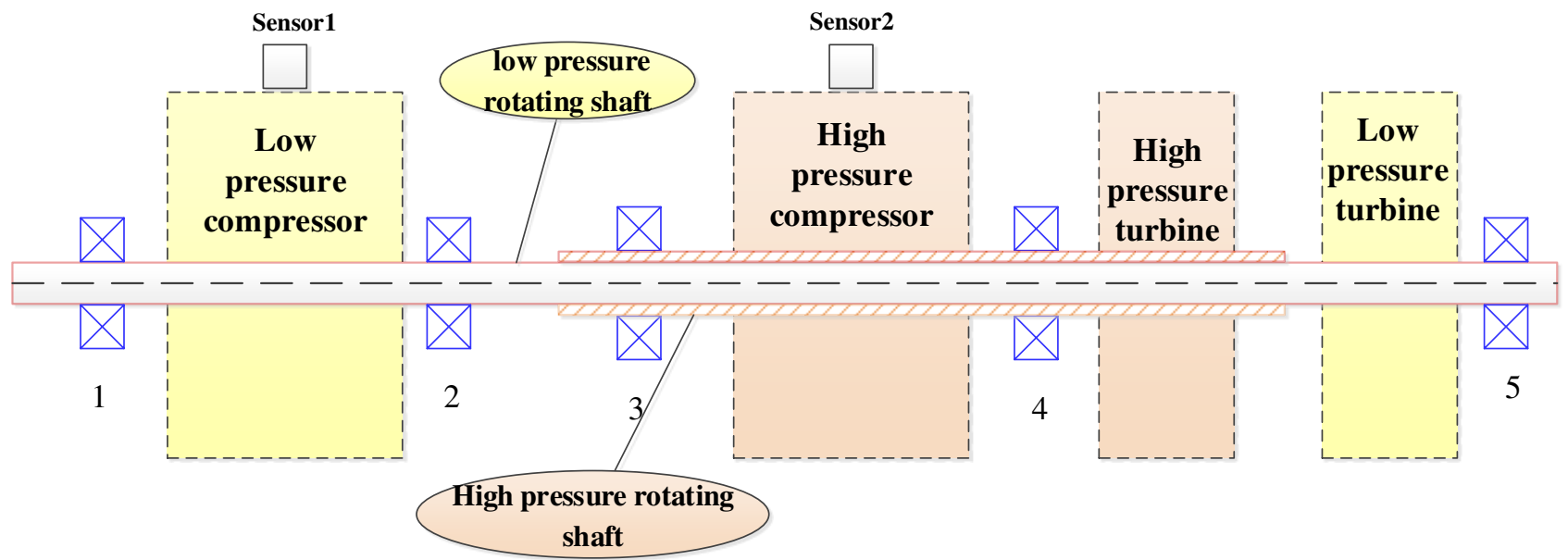

Fig.5 Schematic diagram of gas turbine vibration test

The sampling frequency is $6000 \mathrm{~Hz}$, and the experiment time lasts $8000 \mathrm{~s}$. In the actual operation of the gas turbine rotor system, the time domain statistics in the vibration signals will change with the change of the working condition and state of the gas turbine rotor system. Therefore, the time domain analysis of gas turbine rotor system vibration signals can be used to preliminarily observe and judge the state and trend of rotor system, and further for the gas turbine rotor system fault diagnosis and analysis. The vibration signals collected by the gas turbine sensor are shown in Fig.6, and the effective values of gas turbine vibration signals in time-domain can been obtained. From Fig.6 it can been found that the amplitude of the front measuring point vibration data is large and exceeds the limit in some time periods, by analysis it can be concluded that the faults may occur. For further analysis, the Root Mean Square (RMS) of vibration signals are calculated and shown in Fig.7. 


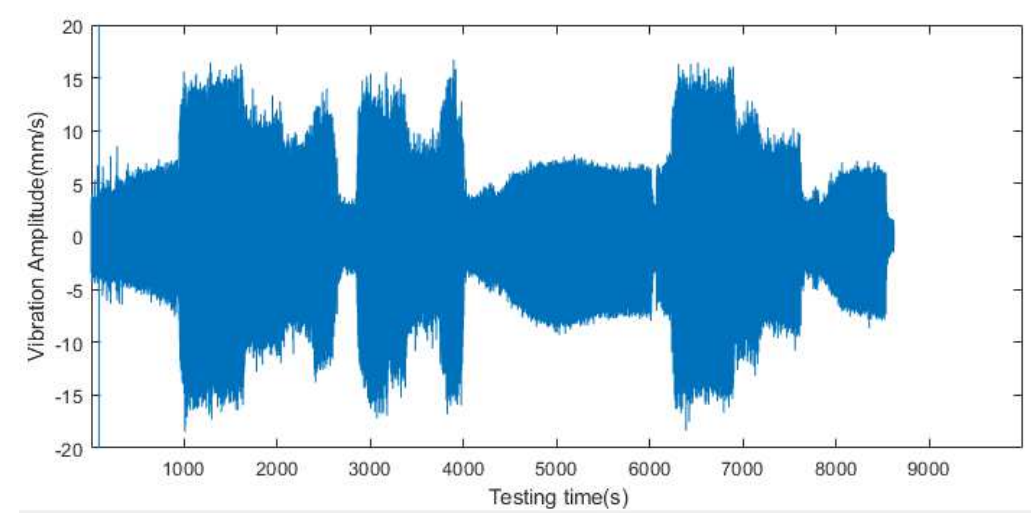

Fig.6 Gas turbine vibration signal at front measuring point

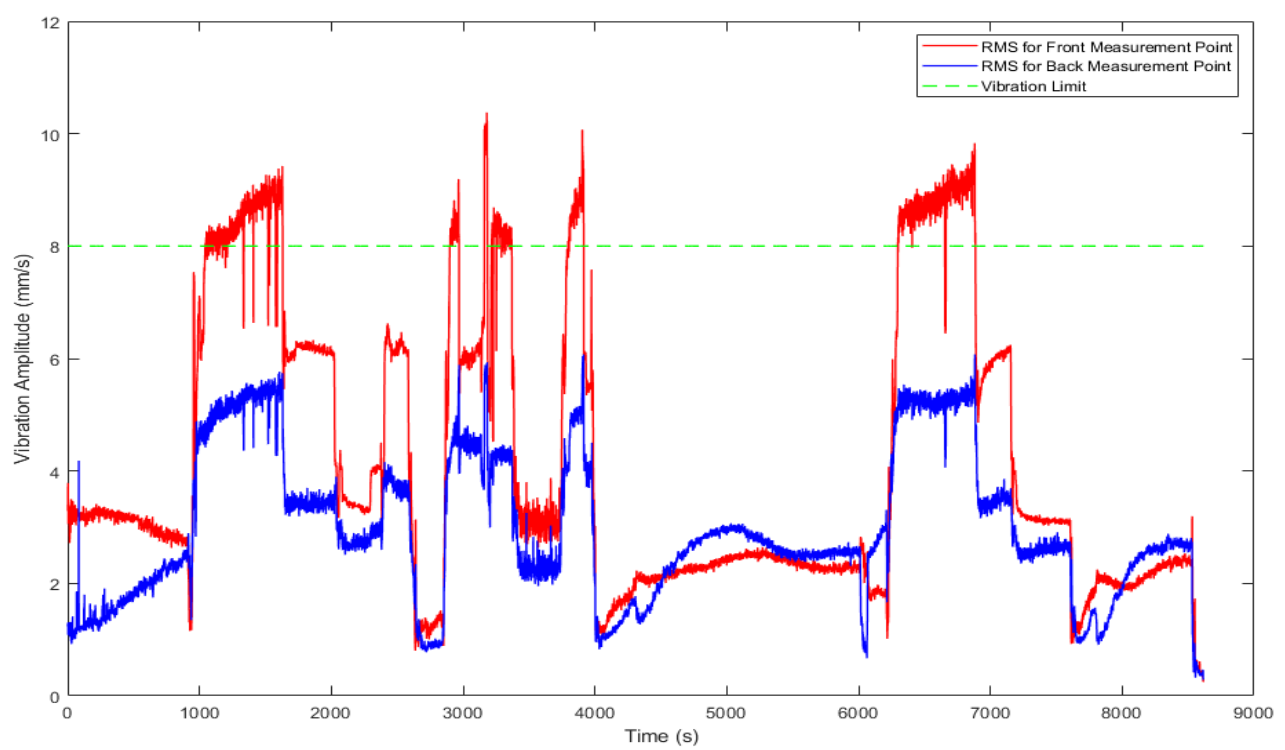

Fig.7 RMS for gas turbine vibration signals

In Fig.7,the high-pressure rotor speed changes continuously under multiple working conditions, the dotted line is the change of the high-pressure rotor frequency, the green line is the vibration limit value, and the red line is the RMS for front measuring point, and the blue line is the RMS for rear measuring point. It can be found from the gas turbine sensor data analysis that the speed has been constantly changing in various working conditions during the experiment. The effective value of the front measuring point vibration signals and the effective value of the rear measuring point vibration signals change with the working conditions. Among them, the effective value of front measuring point vibration signals has the phenomenon of vibration exceeding the limit for several time periods, and the effective value of rear measuring point vibration signals has never exceeded the limit. It can be found that in multiple periods of time, due to the structure and assembly of the gas turbine, the gas turbine stalls due to the rotation of the high-pressure compressor during the speed-up condition, resulting in air flow excitation, further causing temporary imbalance of the high-pressure rotor, resulting in vibration overrun.

Therefore, the pre-measurement vibration data obtained from the gas turbine test bench experiment can be divided into four classes which are normal state, air flow excitation, unbalanced and misalignment, and these test data will be used as the dataset for faults diagnosis. Different classes of vibration signals for gas turbine are shown in Fig.8.

The time-frequency analysis of gas turbine rotor system vibration signals under misalignment and imbalance state are respectively shown in Fig.9.It can be seen from Fig.9(a), the abnormal frequency $(131.5 \mathrm{~Hz})$ is about 1.47 times of the low-voltage rotating frequency $(89.1 \mathrm{~Hz})$, and the frequency is not fixed. A fter preliminary judgment, it is determined as faults by rotor misalignment.In addition to the abnormal frequency associated with excessive vibration, the double frequency component $101 \mathrm{~Hz}$ of low pressure rotor frequency $52 \mathrm{~Hz}$ appears when the high speed drops to slow speed, as shown in Fig.9(b). By analysis, the causes may be caused by the rotor unbalanced. After discussion and consultation with experts, it was preliminarily determined the abnormal frequency and the reasons of vibration exceeded the standard. Through actual faults examine and machine maintenance, the fault type was determined, and the label was given as the experimental sample. 
The rotor misalignment could cause the radial double frequency vibration, axial vibration of the rotor could cause the fatigue damage of the shaft, as shown in Fig.10.If the rotor misalignment lasts for a long time, it will aggravate and cause other more serious faults, it is necessary to be effectively identified.The gas turbine rotor system unbalance will directly lead to excessive vibration of the whole machine, which will cause a series of elements deformed or even damaged. The deformation caused by vibration will produce rub impact, leading to fatigue damage of the rotor or other parts, such as fatigue crack shown in Fig.11.

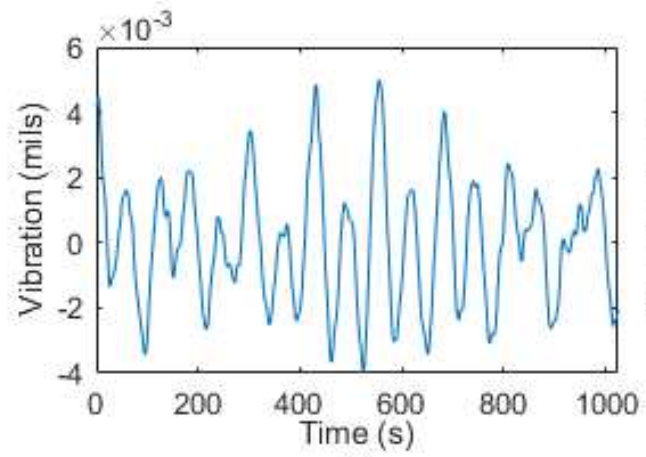

(a)

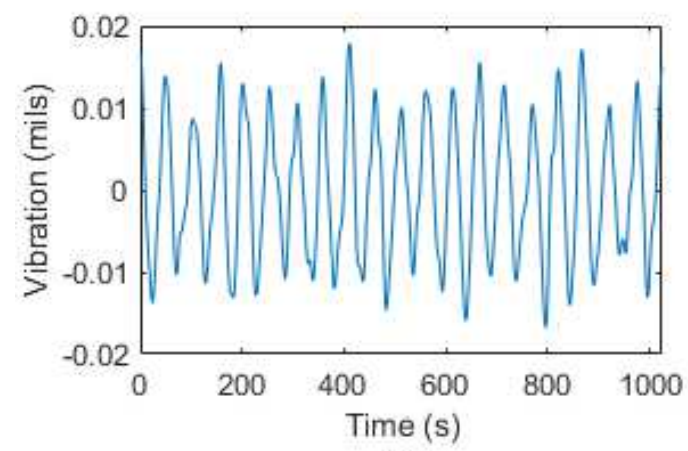

(c)

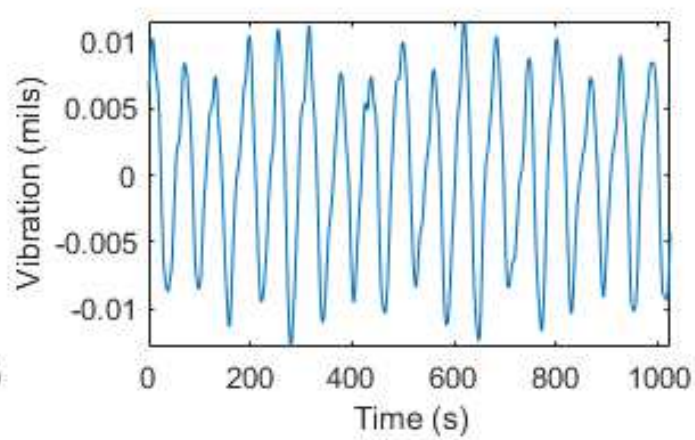

(b)

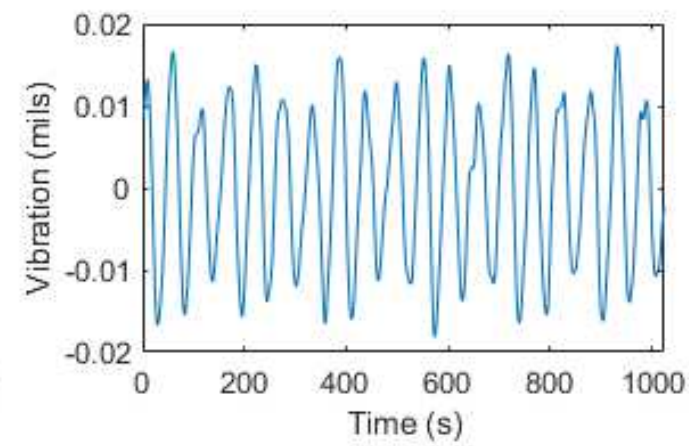

(d)

(a) Normal state (b) Airflow excitation (c) Unbalanced (d) Misalignment

Fig.8 Different vibration signals for gas turbine rotor system faults

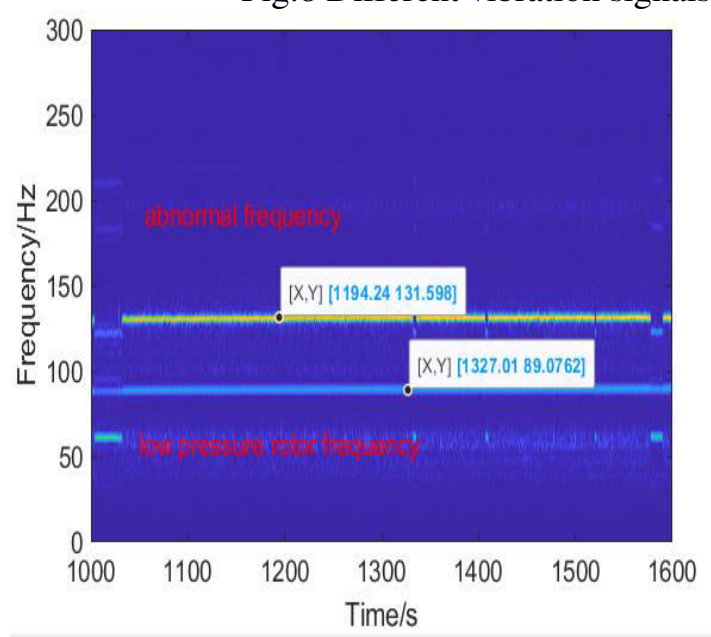

(a)misalignment

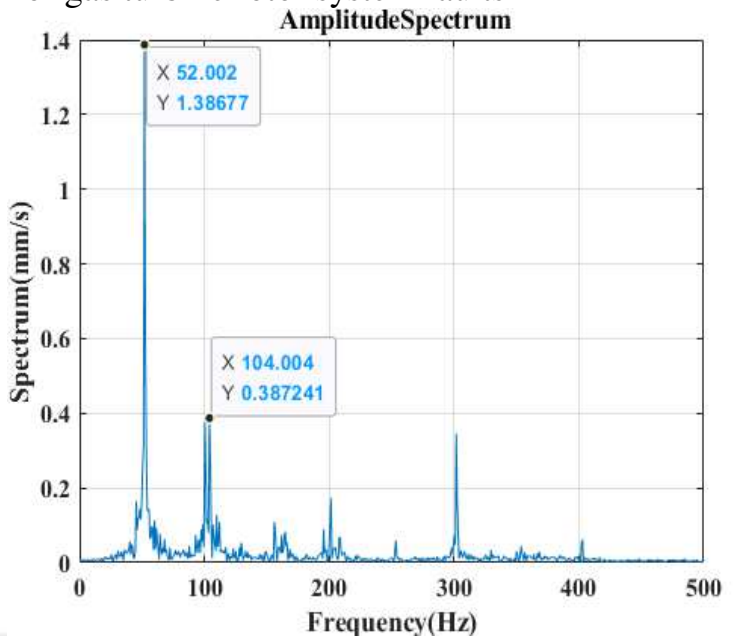

(b)unbalanced

Fig.9 Time-frequency analysis of gas turbine rotor faults 


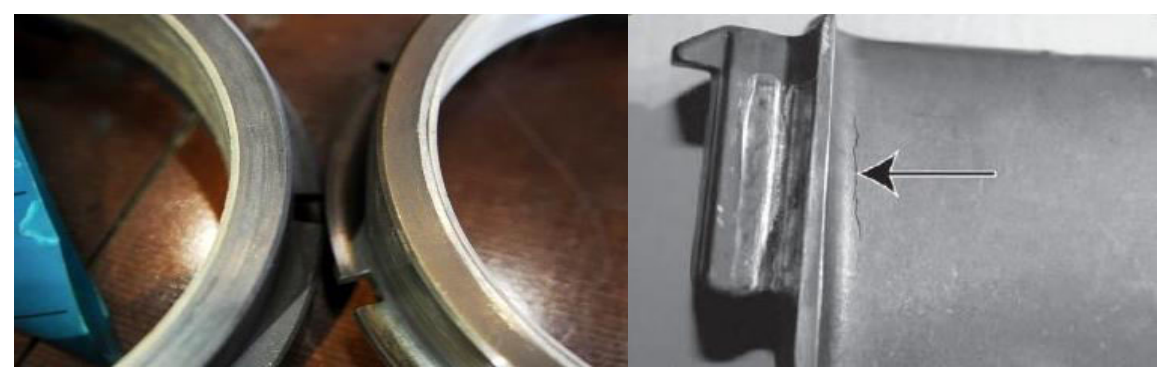

Fig.10 The fatigue damage of the shaft Fig.11 Fatigue crack of blade

\subsection{The training of improved DCGAN}

Because the labeling of fault samples requires a lot of experimental and expert experience, the fault samples are not enough. Therefore, in order to improve the accuracy of fault diagnosis, the proposed method is used to generate fault samples with very high similarity with the original samples to supplement all kinds of samples and make up for the imbalance of samples. Fig. 12 is the flow chart of gas turbine fault data generation and fault diagnosis of gas turbine rotor system. The gas turbine sample set is used as the training data set, the faults samples of each class are 1000. Then the samples are input as to the improved DCGAN to achieve the generated sample. T he generated samples of each class are 1000 .

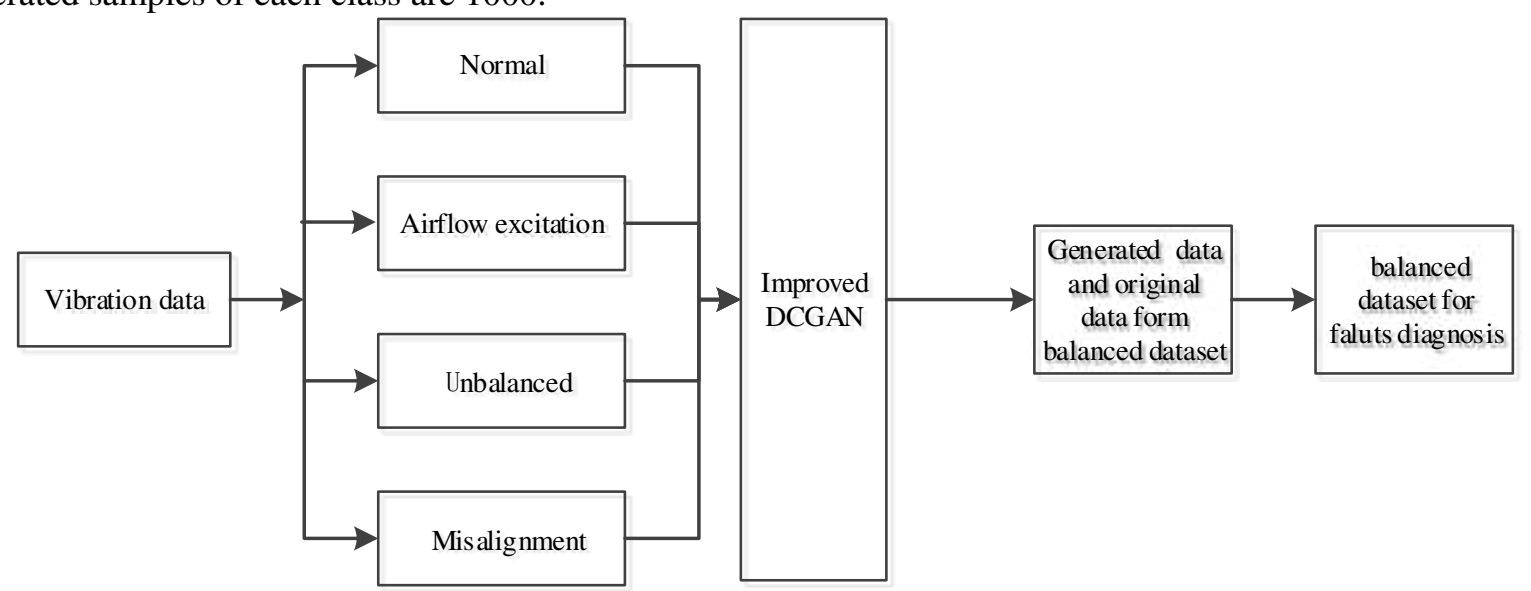

Fig.12 Faults data generation and classification of gas turbine rotor system

The training parameters of the proposed Improved DCGAN network model are set as follows: the batch size is set as 11 , data dimension size is set as 1000 , learning rate is set as 0.000001 , and the number of model training iterations is set to 80000 , generator gradient penalty parameters $\beta$ is set to 0.000001 , discriminator gradient penalty parameter $\lambda$ is set to 10 .The optimization method of generator and discriminator is Adam optimization algorithm. In order to ensure that the generated signals and the real signals have a large degree of similarity and a small error, the MMD should be set as less than 0.125.

In the adversarial training between the generator and discriminator, the generator tries to generate samples which could cheat the discriminator, the discriminator learns to distinguish the difference between the generated samples and the real signals with training.

Fig.13 shows the loss function waveform of generator and discriminator. In adversarial learning between the generator and discriminator, the generated samples increases with iteration, the loss functions gradually changes. At the beginning, the loss of generator keeps smooth, then gradually increases and finally tends to be stable. The loss of the discriminator gradually decreases with the training time, and tends to be stable. After adversarial training, the generator generates higher and higher quality vibration signals. 

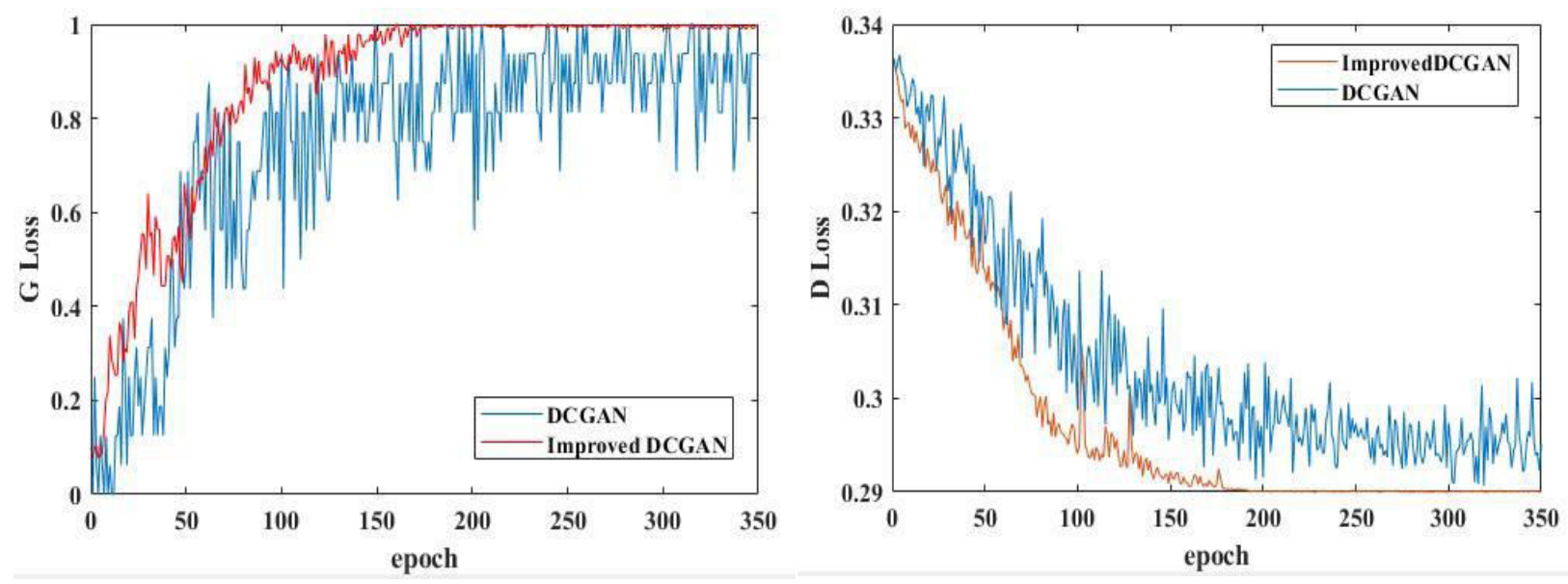

Fig.13 Loss function of generator and discriminator

It can be seen from Fig.13 that compared with DCGAN, the newly added gradient penalty term in the proposed DCGAN made the overall training of the deep convolutional generative adversarial network more stable, and the convergence speed faster.

\subsection{The generated data and results comparison}

The original signals and generated fault sample signals under four states by the proposed method are demonstrated respectively, Fig.14 is the generated sample signals of gas turbine rotor system under normal states,Fig.15 and Fig.16 show the generated sample signals of gas turbine rotor system under airflow excitation state and unbalanced state,Fig. 17 is the generated sample signals of gas turbine rotor system under misalignment state. For comparison the generated fault sample signals by GAN and DCGAN are also displayed.

Meanwhile the original signals and generated signals based on GAN, DCGAN and the improved DCGAN method are analyzed by frequency domain and time-frequency domain spectrum analysis. In order to better verify the similarity between the generated samples and the original samples, spectrum analysis and structural similarity analysis are used for verification.

(1)Spectrum analysis

The similarity between the generated samples and the original samples are analysized by time-domain analysis, Fast Fourier Transform (FFT) spectrum analysis, Short-Time Fourier Transform (STFT) spectrum analysis and Continue Wave Transform (CWT) analysis. It can be seen from the comparison of spectrum signals in normal states in Fig.14that the spectrum of the newly generated sample signals is consistent with the spectrum of real sample signals. It can be seen obviously from FFT and CWT diagram that the samples generated by the proposed method have the highest similarity with the original signals. The generated samples by GAN and DCGAN method are similar to the original samples, but not completely equivalent, and there are small deviations at some peaks.

Fig.15 shows the vibration signals and generated sample signals under airflow excitation from time-domain signals, frequency and time-frequency spectrum. It can be seen from the FFT spectrum diagram of the original signals that there are multiple doubling frequency components in the frequency domain, including fundamental frequency $\mathrm{f}_{0}$, double frequency $2 \mathrm{f}_{0}$, and triple frequency $3 \mathrm{f}_{0}$ respectively. Meanwhile it can be seen from the FFT spectrum diagram of generated samples by the proposed method that these frequency features are very similar to the features of original signals under air flow excitation, which proves that the key features of the samples generated by the proposed method are consistent with that of original samples, and the quality of the generated samples is very good. The samples generated by GAN have no obvious multi-frequency components in FFT diagram, and the samples generated by DCGAN have no obvious multi-frequency components in CWT diagram. By the method the generator can generate new airflow excitation samples with a similar distribution to original samples, which is most consistent with the original signals. With this method, the training samples can be expanded and the training of the classifier can be enhanced to further improve the generalization performance of the model.

Fig.16 shows the generated gas turbine rotor faults sample signals under unbalanced state, it can be seen that the generated samples by the proposed method had higher similarity with original signals than by GAN and DCGAN method.Fig.17 shows the generated gas turbine rotor faults sample signals under misalignment state generated by GAN, DCGAN and improved DCGAN, it can be seen from FFT,STFT and CWT diagram that the frequency of the generated samples by improved DCGAN emerged double frequency component, this indicates the generated gas turbine rotor system unbalanced samples keep consistent with real samples from overall trend and can be used as extended samples of original samples. 

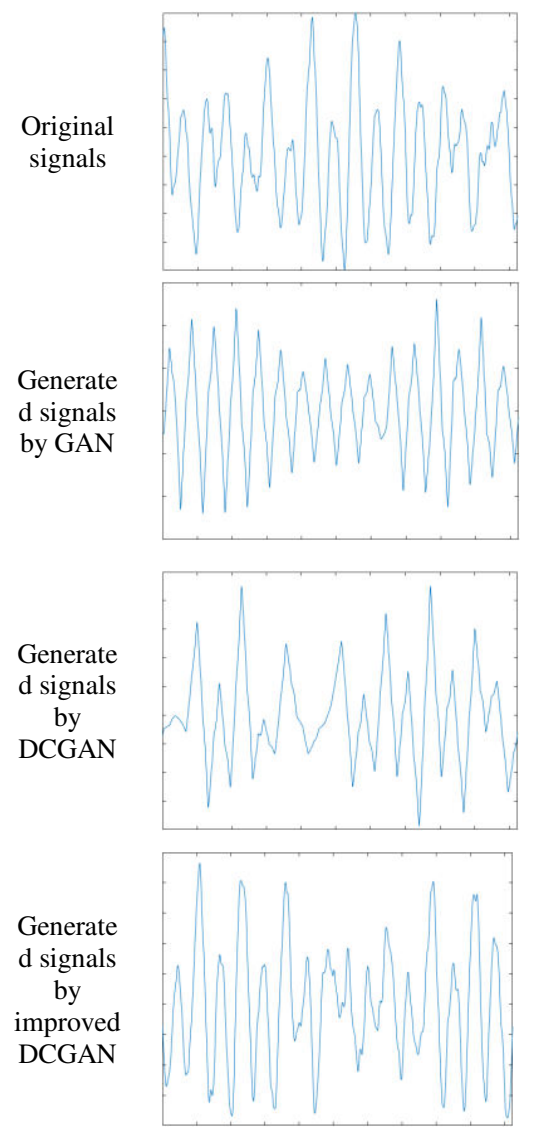

(a)Time domain signals
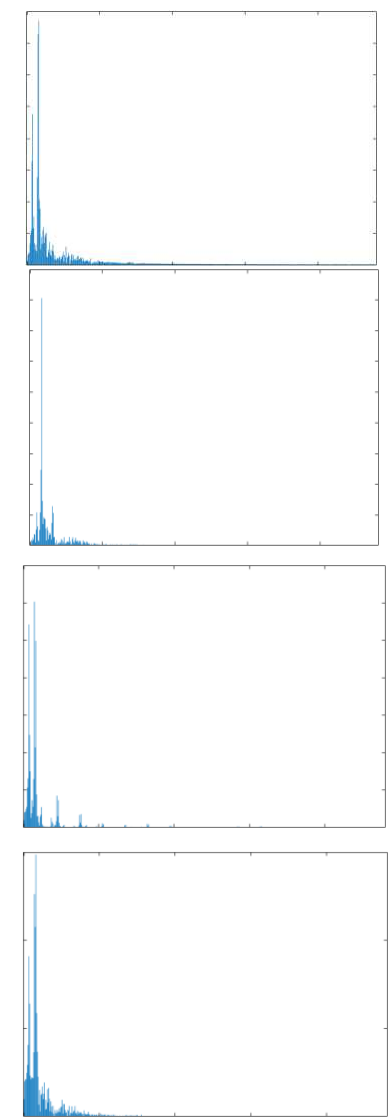

(b) FFT
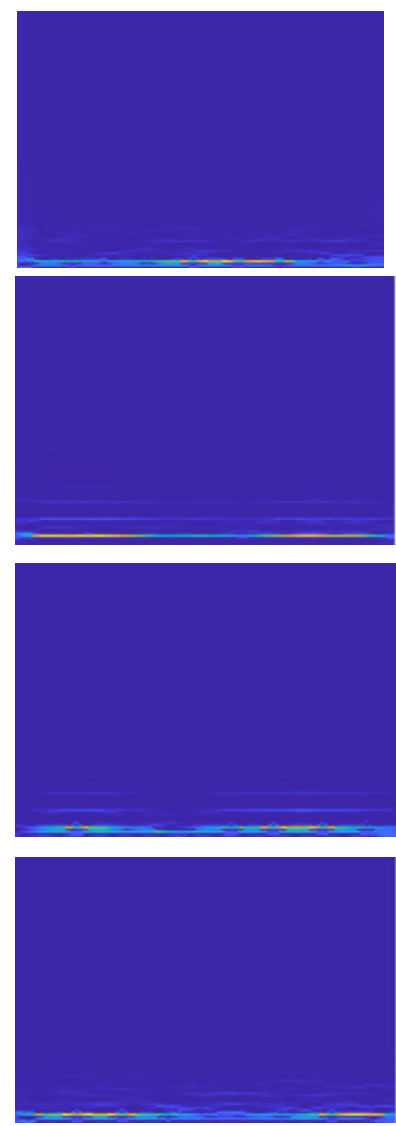

(c)STFT
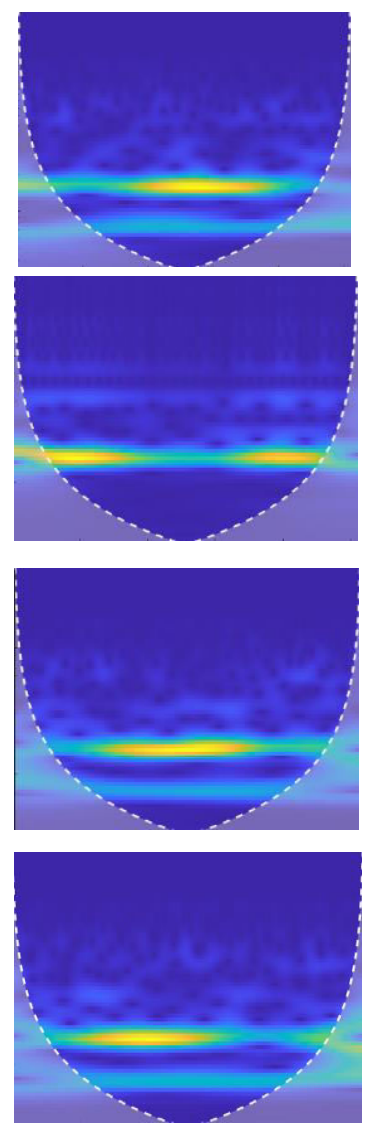

(d) CWT

Fig.14 Signals comparison of time domain and frequency domain of the original signals and the generated signals in normal state

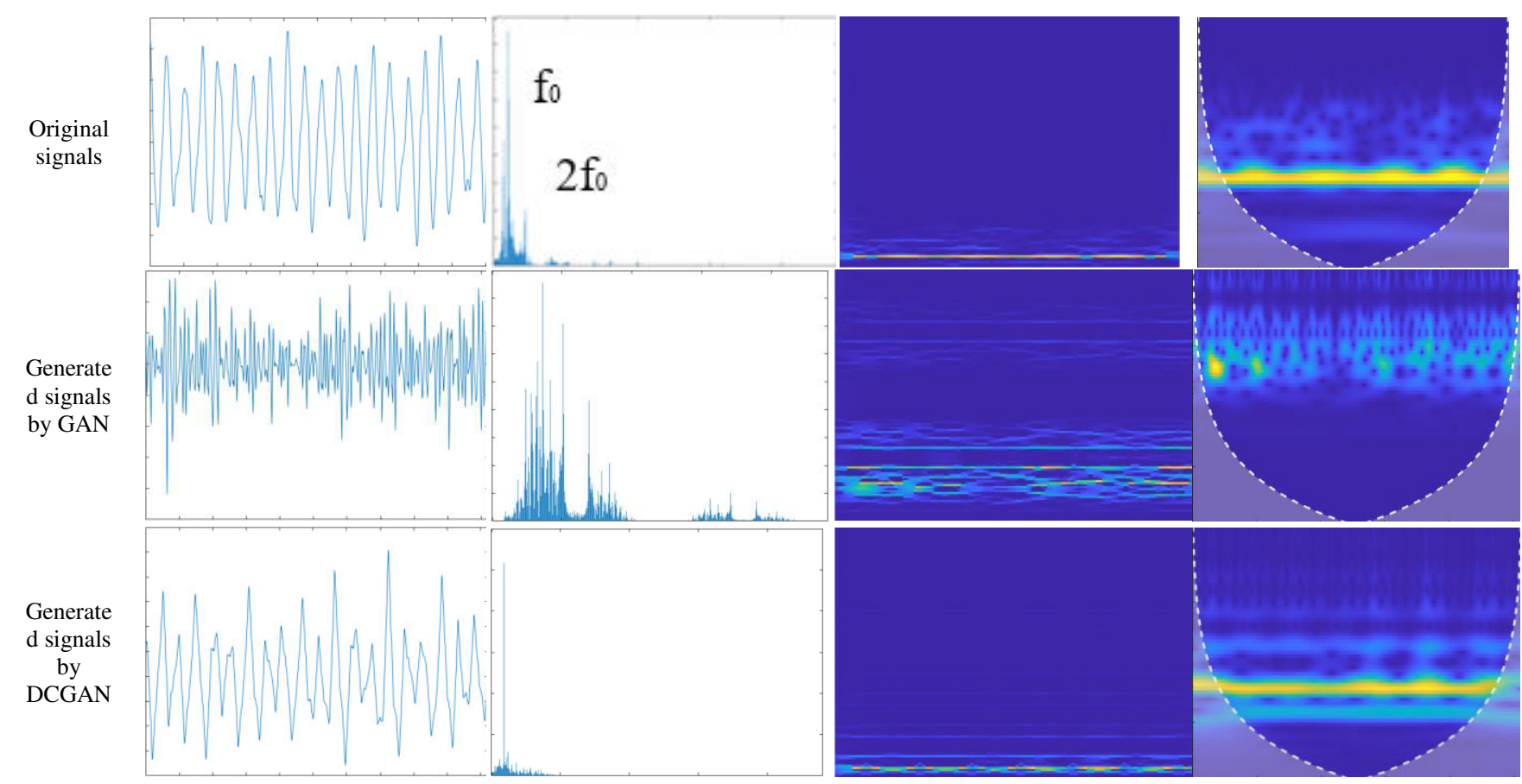




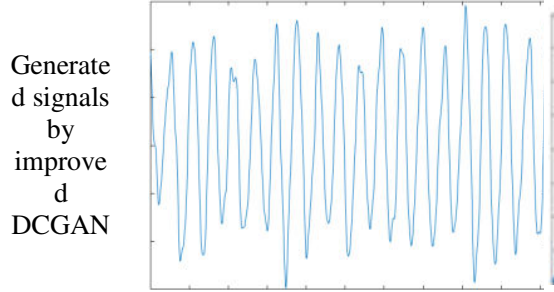

(a)Time domain signals

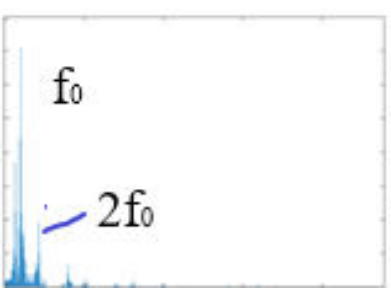

(b) FFT

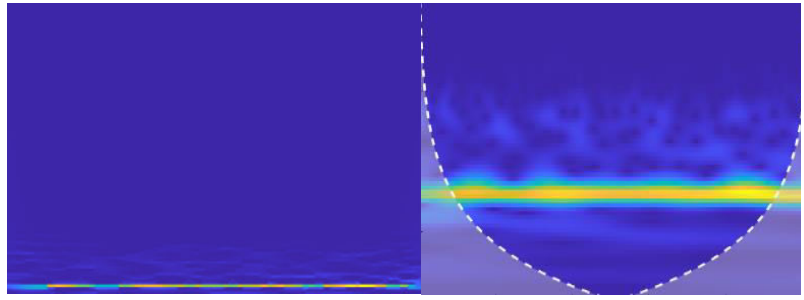

(c)STFT (d) CWT

Fig.15 Signals comparison of time domain and frequency domain of the original signals and the generated signals in airflow excitation state

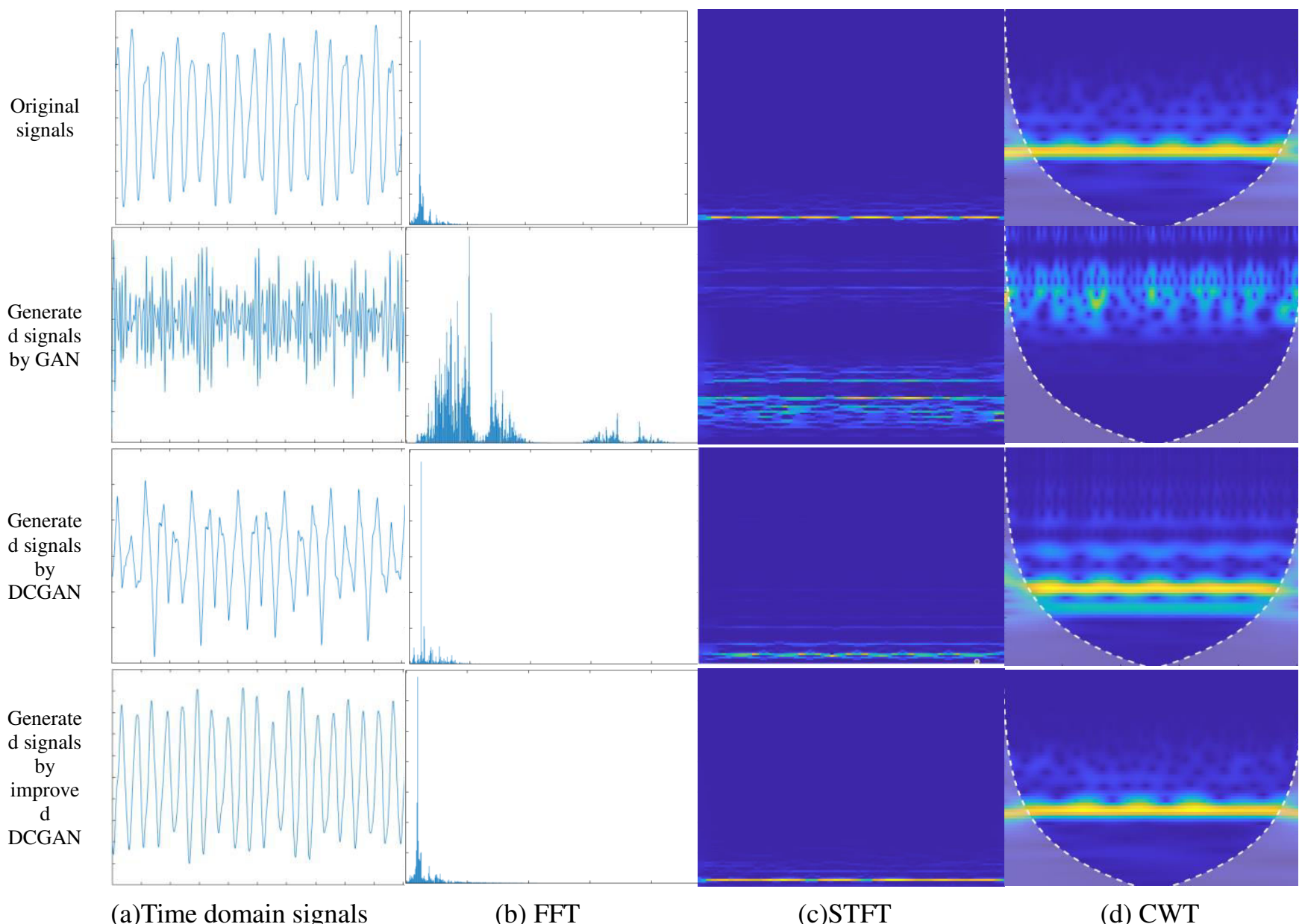

Fig.16 Signals comparison of time domain and frequency domain of the original signals and the generated signals in unbalanced state
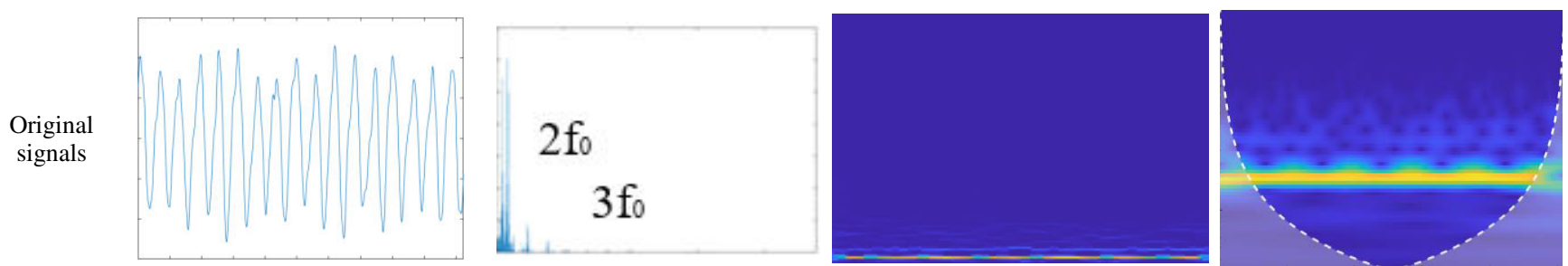


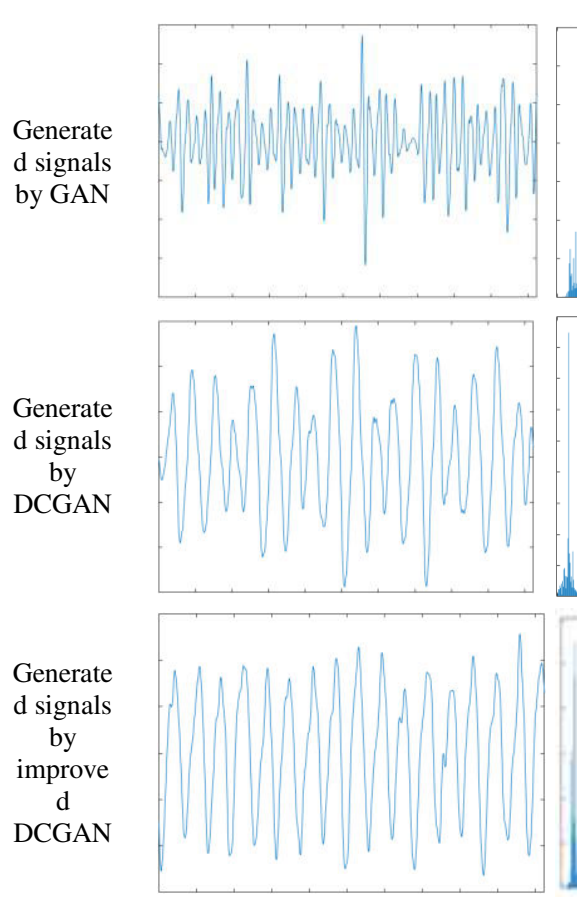

(a)Time domain signals
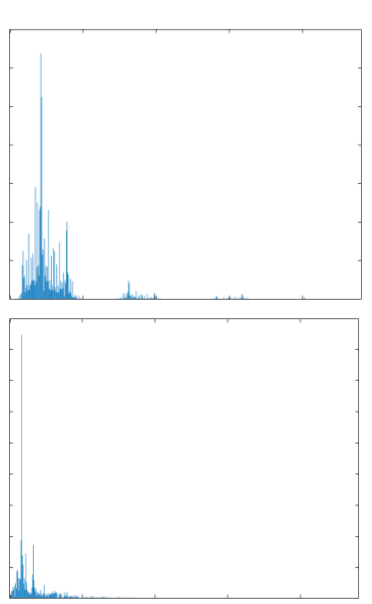

$2 \mathrm{f}_{0}$

$3 \mathrm{f}_{0}$

(b) FFT
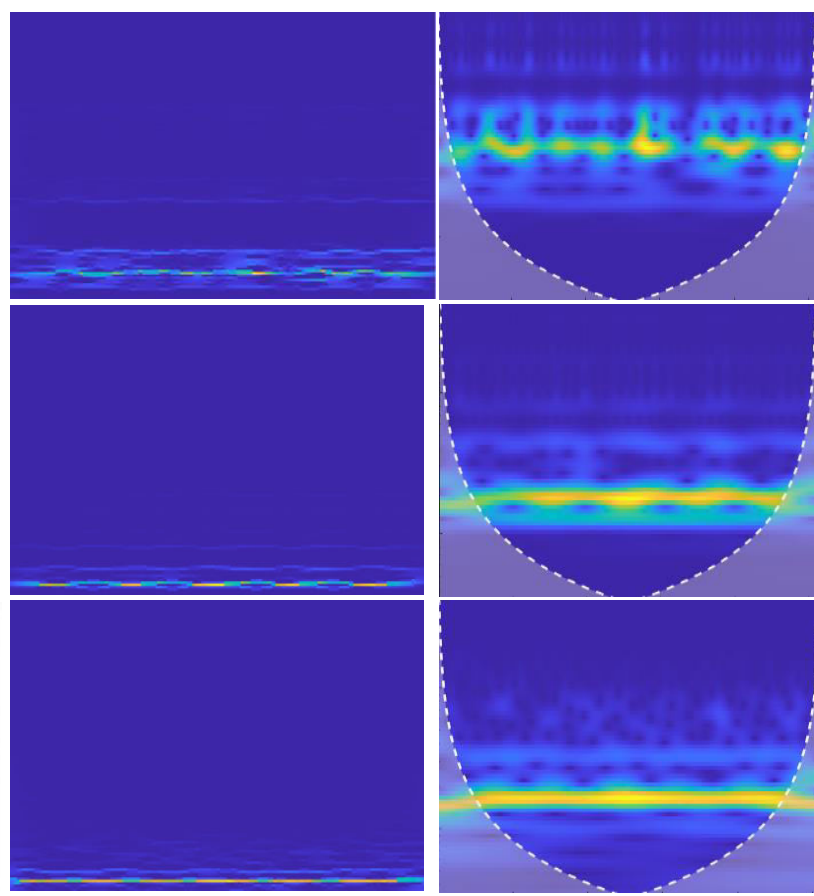

(c)STFT

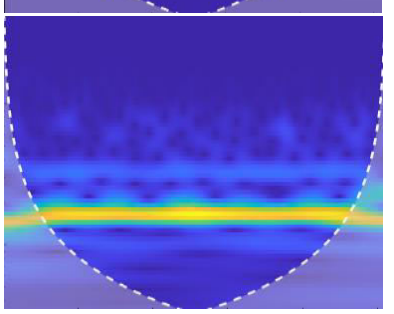

(d) CWT

Fig.17 Signals comparison of time domain and frequency domain of the original signals and the generated signals in misalignment state

(2)Structural similarity analysis

In order to further analyze the accuracy of the generated signals, the SSIM (structural similarity, SSIM) of the obtained FFT,STFT,CWT spectrum images can be used for comparative analysis[48-49].

SSIM (structural similarity, SSIM) evaluates the image quality from three aspects: brightness, contrast and structure, which can be expressed by Eq.(16):

$$
\operatorname{SSIM}(x, y)=[l(x, y)]^{\alpha}[c(x, y)]^{\beta}[s(x, y)]^{\gamma}
$$

Where $x$ represents the measured image and $y$ represents the real image. $l(x, y) 、 c(x, y)$ and $s(x, y)$ respectively represent the brightness term, contrast term and structure term of the image. The calculation process is shown in Eq.(17) to Eq.(19):

$$
\begin{gathered}
l(x, y)=\frac{2 \mu_{x} \mu_{y}+C_{1}}{\mu_{x}^{2}+\mu_{y}^{2}+C_{1}} \\
c(x, y)=\frac{2 \sigma_{x} \sigma_{y}+C_{2}}{\sigma_{x}^{2}+\sigma_{y}^{2}+C_{2}} \\
s(x, y)=\frac{2 \sigma_{x y}+C_{3}}{\sigma_{x} \sigma_{y}+C_{3}}
\end{gathered}
$$

In which $\mu_{x}$ and $\mu_{y}$ represent the mean value of the measured image and the real image respectively, $\sigma_{x}^{2}$ and $\sigma_{y}^{2}$ respectively represent the variance between the measured image and the real image, $\sigma_{x} \sigma_{y}$ represents the covariance between the measured image and the real image. $C_{1} 、 C_{2}$ and $C_{3}$ are the constant term to avoid the denominator being zero, and the relationship between the three are $C_{1}=\left(K_{1} \times L\right)^{2}, C_{2}=\left(K_{2} \times L\right)^{2}$ and $C_{3}=C_{2} / 2$,in which $K_{1}=0.01, K_{2}=0.03, L=255$. The simplified equation of SSIM is converted to Eq. (20): 


$$
\operatorname{SSIM}(x, y)=\frac{\left(2 \mu_{x} \mu_{y}+C_{1}\right)\left(2 \sigma_{x} \sigma_{y}+C_{2}\right)}{\left(\mu_{x}^{2}+\mu_{y}^{2}+C_{1}\right)\left(\sigma_{x}^{2}+\sigma_{y}^{2}+C_{2}\right)}
$$

The value range of SSIM is between zero and one. The larger of SSIM values, the more similar the two images are, and the better quality of the measured image. According to SSIM, the SSIM of FFT, STFT diagram and CWT diagram of generated signals are calculated and compared with SSIM of the real signals spectrum. The results are shown in Table 2.

Table2 SSIM Comparisons on between generated signals and real signals under different states

\begin{tabular}{|l|l|l|l|l|l|l|}
\hline Faults & \multicolumn{3}{|c|}{ STFT } & \multicolumn{3}{c|}{ CWT } \\
\hline & GAN & DCGAN & Improved DCGAN & GAN & DCGAN & Improved DCGAN \\
\hline Normal & 0.8699 & 0.9502 & 0.9937 & 0.7884 & 0.9456 & 0.9660 \\
\hline Airflow excitation & 0.8522 & 0.9482 & 0.9937 & 0.7874 & 0.9368 & 0.9885 \\
\hline Unbalanced & 0.8576 & 0.9403 & 0.9955 & 0.7959 & 0.9345 & 0.9683 \\
\hline Misalignment & 0.8412 & 0.9214 & 0.9944 & 0.7976 & 0.9421 & 0.9683 \\
\hline
\end{tabular}

From Table2 it can be concluded that the SSIM of generated samples signals by DCGAN is larger than the SSIM of generated samples signals by GAN, and the structural similarity of the four generated fault samples signals generated by the improved DCGAN method is strongest, which meets the requirements of samples required for fault diagnosis.

By combining the powerful feature extraction of CNN with data generation ability of DCGAN, the improved DCGAN method was proposed and verified in gas turbine rotor system test experiments, the result demonstrated that it can generate effective samples, and solve the problem of difficulty to extract gas turbine rotor fault features under the condition of unbalanced and small samples.

\subsection{Classification model and results}

After new samples are generated, the original samples and generated faults sample form a new dataset. The gas turbine sample dataset is divided into training dataset and test dataset. Each dataset have 4000 samples for faults diagnosis, in which every class faults have 1000 samples. In order to examine the superiority of the proposed method, several fault diagnosis methods including Support Vector Machine(SVM),Deep Belief Network(DBN)[50],two dimensional Convolutional Neural Network with Short-Time Fourier Transform of the signals as input (STFT-CNN),WDCNN,Long Short-Term Memory(LSTM)[50] are adopted for diagnosis classification comparison of the target data.SVM is proposed on the basis of statistical learning theory and used in mechanical fault diagnosis because of its faster training convergence rate, stronger generalization ability. DBN saves the process of manually extracting features and fuses feature extraction and classification together. STFT-CNN adopts the Short-time Fourier Transform (STFT) of the obtained sample signals as input and twodimensional convolutional neural network is used for faults classification and analysis. WDCNN is more suitable for one-dimensional vibration signals with high classification accuracy, and the parameter settings of WDCNN are the same as those in Table1. LSTM has strong adaptability for time series data and is widely used in prediction and classification.

Table3 shows the specific classification results of each fault by different diagnosis methods. When SVM is used to classify new samples, the classification accuracy of normal state, airflow excitation, unbalanced and misalignment is $92.50 \%, 94.60 \%, 90.30 \%, 91.30 \%$ respectively, and the average classification accuracy is $92.18 \%$. The average classification accuracy of DBN and STFT-CNN improved to 98.03\% and 98.08\%. When WDCNN is used to classify new samples, the classification accuracy for normal state, airflow excitation, unbalanced and misalignment is $99.80 \%, 98.1 \%, 98.30 \%$ and $98.40 \%$ respectively, and the average classification accuracy is 98.65\%. From the classification results, it can be seen that WDCNN is suitable for gas turbine rotor faults classification, and then WDCNN is chose as the classification model for gas turbine rotor faults classification. The proposed improved DCGAN model generates higher quality generated data, then the new dataset can be used for further faults classification, and the WDCNN accurately learns the data distribution and features of different states of gas turbine, and can provide higher diagnosis accuracy for gas turbine rotor system.

Table3 Comparison of different diagnostic models

\begin{tabular}{cccccc}
\hline & \multicolumn{4}{c}{ Fault Diagnosis Accuracy } \\
\cline { 2 - 6 } Method & Normal & $\begin{array}{c}\text { Airflow } \\
\text { excitation }\end{array}$ & Unbalanced & misalignment & Average \\
\hline DBN & $98.90 \%$ & $98.40 \%$ & $97.80 \%$ & $97.00 \%$ & $98.03 \%$ \\
SVM & $92.50 \%$ & $94.60 \%$ & $90.30 \%$ & $91.30 \%$ & $92.18 \%$
\end{tabular}




\begin{tabular}{cccccc} 
STFT-CNN & $97.30 \%$ & $97.80 \%$ & $98.40 \%$ & $98.80 \%$ & $98.08 \%$ \\
WDCNN & $99.80 \%$ & $98.10 \%$ & $98.30 \%$ & $98.40 \%$ & $98.65 \%$ \\
LSTM & $96.80 \%$ & $98.60 \%$ & $93.20 \%$ & $98.70 \%$ & $96.83 \%$ \\
\hline
\end{tabular}

\subsection{Results visualization comparison}

In order to verify the performance of the proposed method, the gas turbine dataset without generated samples、 with generated samples by GAN 、 with generated samples by DCGAN are also selected to train the CNN model and classify the faults.Fig.18 displayed the classification results of these methods. It can be concluded that the classification accuracy of the proposed improved DCGAN method obviously increased comparing with the dataset without generated samples. Meanwhile the classification accuracy of the proposed improved DCGAN method are higher than the classification accuracy of GAN and DCGAN method.

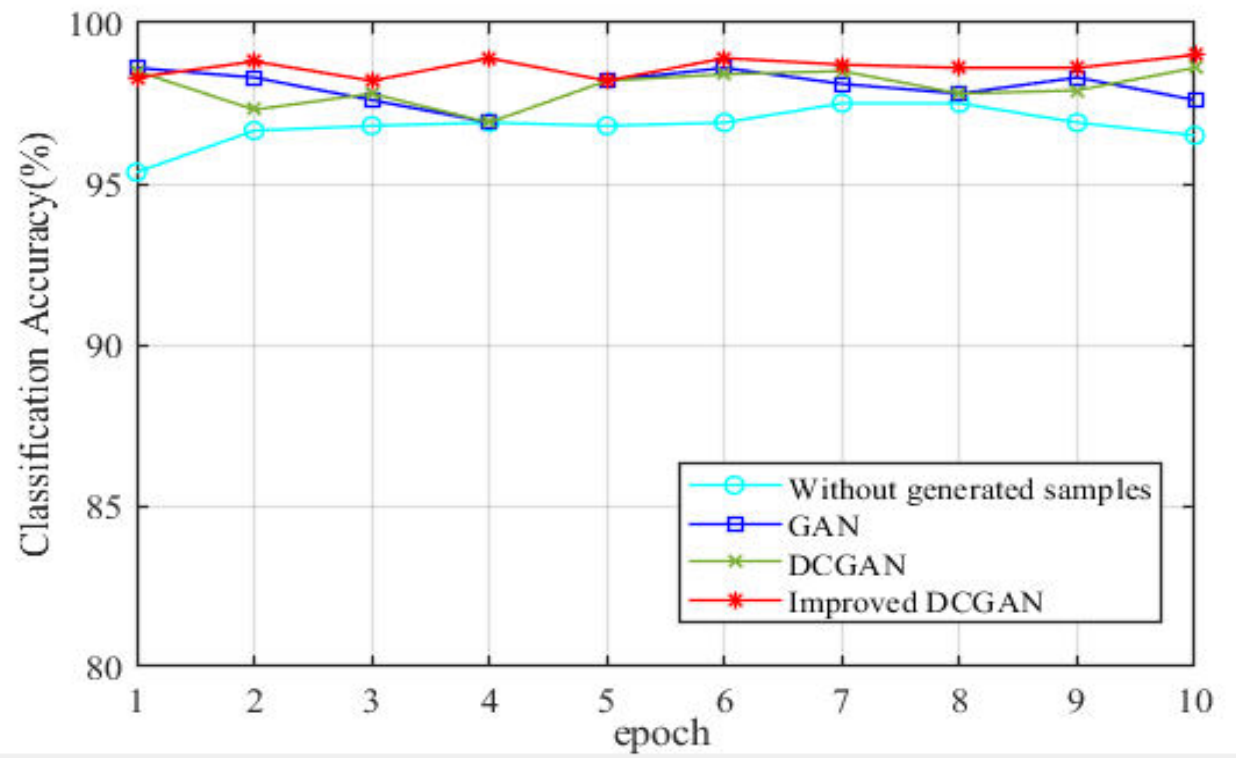

Fig.18 Classification results comparisons

For the sake of display the classification results after implementing the faults dataset by these methods, the visualized t-distributed Stochastic Neighbor Embedding (t-SNE)diagram[52]are shown in Fig.19. It can be seen intuitively in Fig.19 (a) the original samples without generated samples are mostly classified, some fault samples of different kinds are gathered together and not well separated, and some faults are misclassified. In Fig.19 (b) and Fig.19(c) the classification results improved. In Fig.19 (d) the classification results of the proposed method are the best, with most samples are classified correctly.

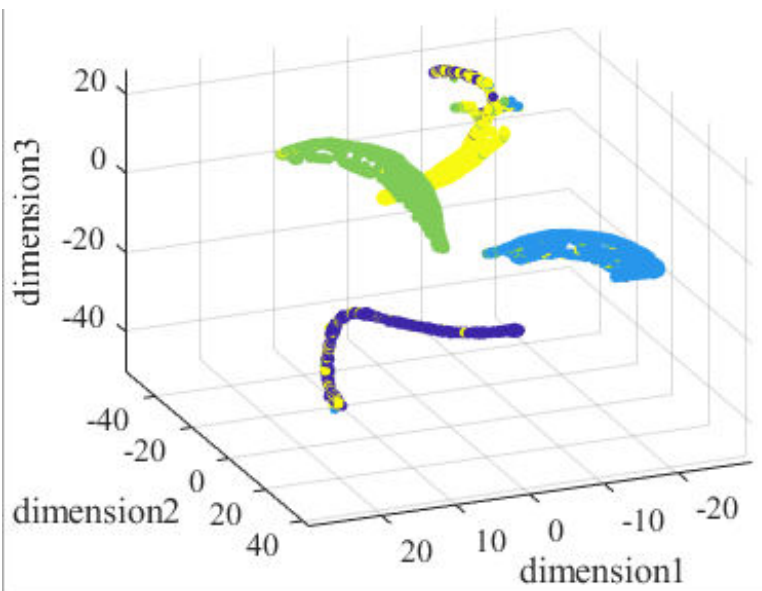

(a)Without generated samples

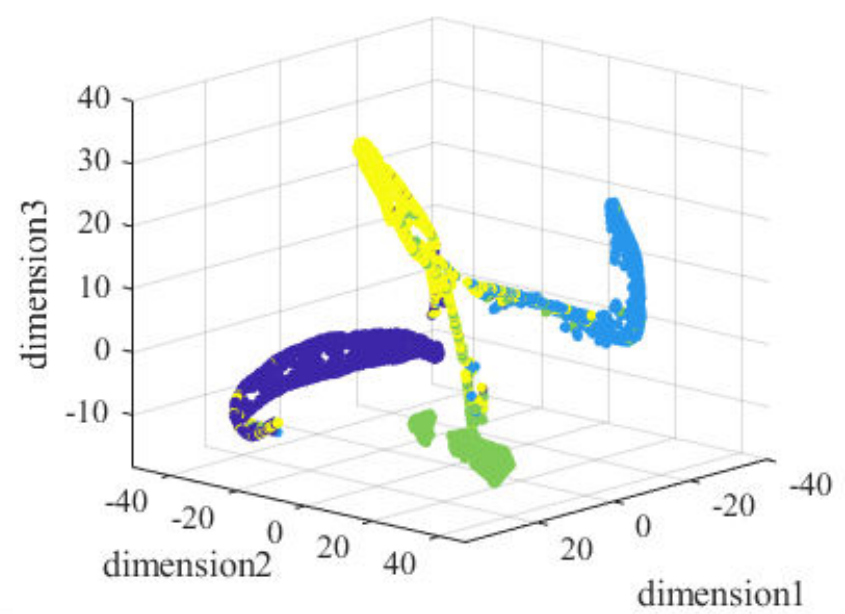

(b) Including generated samples by GAN 

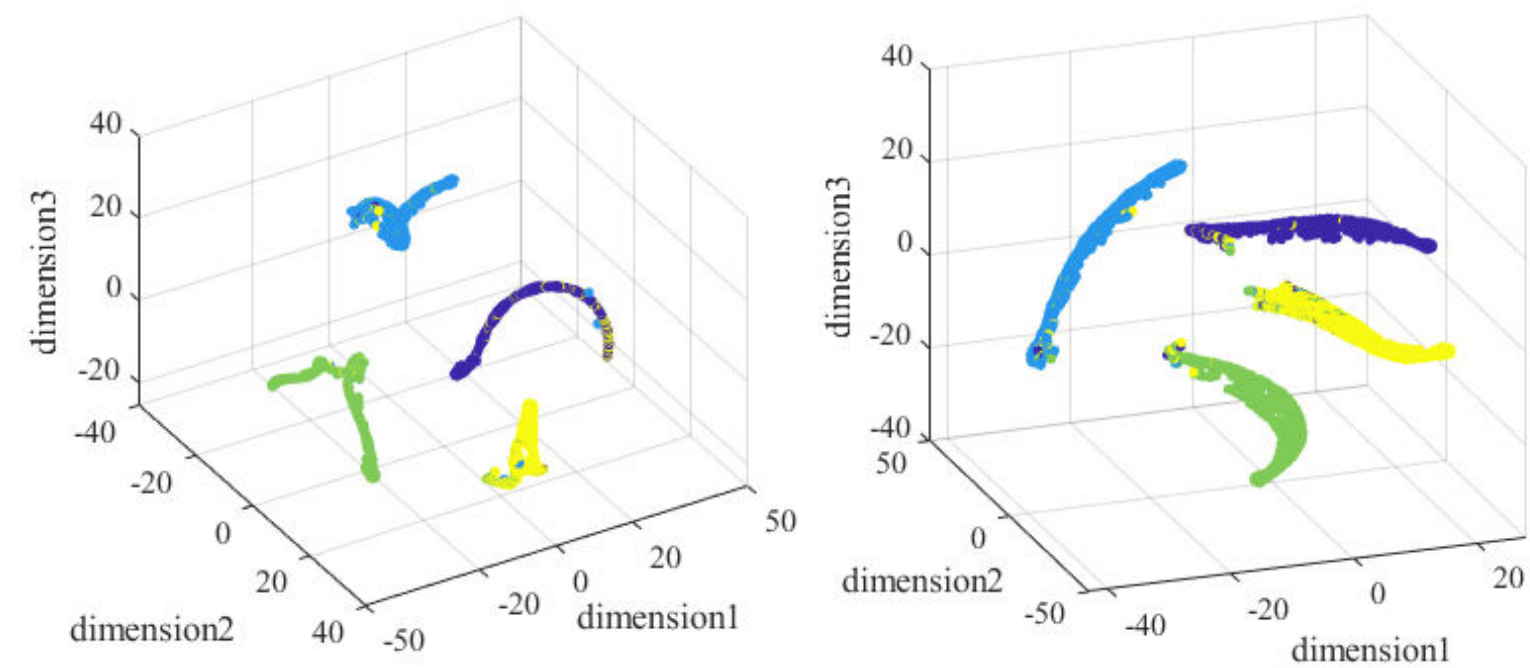

(c)Including generated samples by DCGAN

(d) Including generated samples by improved DCGAN

Fig.19Accuracy comparisons with different methods

\section{Conclusion}

In this paper, an data augmentation method based on improved Deep Convolution Generation Adversarial Network for gas turbine rotor system fault diagnosis is proposed. This method utilizes the powerful feature extraction and data generation ability of GAN to generate effective faults samples for faults diagnosis. The main highlights are sum up as follows:

Firstly, on the base of Deep Convolution Generation Adversarial Network, an improved DCGAN method combining the data generation ability of DCGAN with the feature extraction ability of WDCNN was proposed, and a new signal generator more suitable for gas turbine rotor faults signals is constructed.

Secondly, gradient penalty was adopted to adaptively optimize the loss function according to different gas turbine rotor system faults under unknown conditions.

Finally, the effectiveness of the method is verified by gas turbine rotor system faults experiments. The results indicated that the classification accuracy improved greatly by the proposed method comparing with GAN method and DCGAN method, and the method can generate effective faults samples to solve imbalance of fault samples.

To sum up, the proposed method effectively supplement fault sample, solves the problem of small samples and unbalanced samples, improves the fault diagnosis accuracy, and provides an effective method and data supply for fault diagnosis of gas turbine rotor system.

Acknowledgments: This work was supported by the National Natural Science Foundation of China (No.51975058).

Conflicts of Interest: The authors declare no conflict of interest.

\section{References}

[1]Zaidan M A, Relan R , Mills A R , et al. Prognostics of gas turbine engine: An integrated approach[J]. Expert Systems with Applications, 2015, 42(22):8472-8483.

[2]Ahmadi P , Saidi M H , Dincer I . Performance Assessment of a Hybrid Solid Oxide Fuel Cell-Gas Turbine Combined Heat and Power System[M]. Progress in Exergy, Energy, and the Environment. Springer International Publishing, 2014.

[3]Palm é T,FastM,ThernM.Gas turbine sensor validation through classification with artificial neural networks[J].Applied Energy,2011,88(11):3898-3904. 
[4]Xia F,Zhang H,Peng D,Li H,Su Y.Turbine fault diagnosis based on fuzzy theory and SVM[C]. In: Artificial intelligence and computational intelligence.Springer;2009.p.668-676.

[5]Mirhosseini A M , Nazari S A , Pour A M , et al. Probabilistic failure analysis of hot gas path in a heavy-duty gas turbine using Bayesian networks[J]. International journal of systems assurance engineering and management, 2019, 10(5):1173-1185.

[6]GholamrezaeiM,GhorbanianK.Application of integrated fuzzy logic and neural networks to the performance prediction of axial compressors.Proc Inst Mech Eng,PartA:J Power Energy,2015.0957650915596877.

[7] Chen J , Xu C , Ying Y , et al. Gas-path Component Fault Diagnosis for Gas Turbine Engine: A Review[C]. 2019 Prognostics and System Health Management Conference (PHM-Qingdao). 2019.

[8]Khaljani M , Saray R K , Bahlouli K . Comprehensive analysis of energy, exergy and exergo-economic of cogeneration of heat and power in a combined gas turbine and organic Rankine cycle[J]. Energy Conversion \& Management, 2015, 97:154-165.

[9]M, Amozegar, K, et al. An ensemble of dynamic neural network identifiers for fault detection and isolation of gas turbine engines[J]. Neural Networks, 2016, 76:106-121.

[10]Buonomano, Annamaria, Calise, et al. Hybrid solid oxide fuel cells-gas turbine systems for combined heat and power: A review[J]. Applied energy, 2015,156(OCT.15):32-85.

[11]Zhang $\mathrm{J}, \mathrm{Ma} \mathrm{W}$, Lin $\mathrm{J}$, et al. Fault diagnosis approach for rotating machinery based on dynamic model and computational intelligence[J]. Measurement, 2015, 59:73-87.

[12] Shao $\mathrm{H}$, Jiang $\mathrm{H}$, Zhao $\mathrm{H}$, et al. A novel deep autoencoder feature learning method for rotating machinery fault diagnosis[J]. Mechanical Systems and Signal Processing, 2017,95:187-204.

[13] Sun H, He Z, Zi Y, et al. Multiwavelet transform and its applications in mechanical fault diagnosis-a review[J]. Mechanical Systems and Signal Processing,2014,43(1-2):1-24.

[14] Cabal-Yepez E, Garcia-Ramirez A G, Romero-Troncoso R J, et al. Reconfigurable monitoring system for time-frequency analysis on industrial equipment through STFT and DWT[J]. IEEE Transactions on Industrial Informatics, 2013,9(2):760-771.

[15]MohammadiE,Montazeri-GhM.Afuzzy-basedgasturbinefaultdetectionandidentificationsystemforfullandpart-loa dperformancedeterioration.AerospSciTechnol2015;46:82-93.

[16]Hanachi H , Mechefske C , Liu J , et al. Performance-Based Gas Turbine Health Monitoring, Diagnostics, and Prognostics: A Survey[J]. IEEE Transactions on Reliability, 2018:1340-1363.

[17] Ma A, Sm A, Hs A, et al.Failure and Metallurgical Defects Analysis of IN-738LC Gas Turbine Blades[J]. Engineering Failure Analysis, 2021.https://doi.org/10.1016/j.engfailanal.2021.105213.

[18]Tahan M , Muhammad M , Karim Z A . A multi-nets ANN model for real-time performance-based automatic fault diagnosis of industrial gas turbine engines[J]. Journal of the Brazilian Society of Mechanical Sciences \& Engineering, 2017, 39(7):2865-2876.

[19]Saidi L, Ali J B, Fnaiech F. Application of higher order spectral features and support vector machines for bearing faults classification[J]. ISA transactions,2015,54:193-206.

[20]GaymeD,MenonS,BallC,MukavetzD,NwadiogbuE.Faultdiagnosisingasturbineenginesusingfuzzylogic.In:2003I EEEinternationalconferenceonsystems,manandcybernetics.IEEE;2003.p.3756-62.

[21] Wang H, Du W. A new K -means singular value decomposition method based on self-adaptive matching pursuit and its application in fault diagnosis of rolling bearing weak fault. International Journal of Distributed Sensor Networks,2020,16(5):1-12.

[22] Mbr A, Aha B , Ak A, et al. Gas turbine monitoring using neural network dynamic nonlinear autoregressive with external exogenous input modelling[J]. Mathematics and Computers in Simulation, 2021, 179:23-47. 
[23] Rao A , Satish T N , Nambiar A S , et al. Challenges in Engine Health Monitoring Instrumentation During Developmental Testing of Gas Turbine Engines[J]. 2021.

[24] Yang X , Bai M , Liu J , et al. Gas path fault diagnosis for gas turbine group based on deep transfer learning[J]. Measurement, 2021(5):109631.

[25]Karri S P K , Chakraborty D , Chatterjee J . Transfer learning based classification of optical coherence tomography images with diabetic macular edema and dry age-related macular degeneration[J]. Biomedical Optics Express, 2017, 8(2):579.

[26]Xiao D , Huang Y , Qin C , et al. Transfer learning with convolutional neural networks for small sample size problem in machinery fault diagnosis[J]. ARCHIVE Proceedings of the Institution of Mechanical Engineers Part C Journal of Mechanical Engineering Science 1989-1996 (vols 203-210), 2019, 233(14):095440621984038.

[27]ZHAO Yukai, XU Gaowei, LIU Min .Method for fault diagnosis of bearing based on transfer learning with VGG16 model

[28] Liu S , Jiang H , Wu Z , et al. Data synthesis using deep feature enhanced generative adversarial networks for rolling bearing imbalanced fault diagnosis[J]. Mechanical Systems and Signal Processing, 2022, 163(Jan.):108139.

[29] Bharti V , Biswas B , Shukla K K . EMOCGAN: a novel evolutionary multiobjective cyclic generative adversarial network and its application to unpaired image translation[J]. Neural Computing and Applications, 2021:1-15.

[30]J Luo, J Huang, Li H . A case study of conditional deep convolutional generative adversarial networks in machine fault diagnosis[J]. Journal of Intelligent Manufacturing, 2021, 32.

https://doi.org/10.1007/s10845-020-01579-w.

[31]Rugen Wang, Shaohui Zhang, Zhuyun Chen, Weihua Li. Enhanced generative adversarial network for extremely imbalanced fault diagnosis of rotating machine[J]. Measurement, 180(2021),109467.

[32] Viola J , Chen Y Q , Wang J . FaultFace: Deep Convolutional Generative Adversarial Network (DCGAN)

based Ball-Bearing Failure Detection Method[J]. InformationSciences,542(2021):195-211. https://doi.org/10.1016 /j.ins.2020.06.060.

[33]Qi Li, Liang Chen, Changqing Shen, Bingru Yang. Enhanced generative adversarial networks for fault diagnosis of rotating machinery with imbalanced data[J]. Measurement Science and Technology, 2019, 30(11).

[34] Wu Q , Chen Y, Meng J . DCGAN Based Data Augmentation for Tomato Leaf Disease Identification[J]. IEEE Access, 2020, PP(99):1-1.

[35]Fuan W,Hongkai J,Haidong S,et al.An adaptive deep convolutional neural network for rolling bearing fault diagnosis[J].Measurement Science and Technology,2017,28(9):095005.

[36] Dewi C, Chen R C , Liu Y T , et al. Synthetic Data generation using DCGAN for improved traffic sign recognition[J]. Neural Computing and Applications, 2021:1-16.

[37]JieLi,BoyuZhao,KaiWu,ZhichengDong,XueruiZhangandZhihaoZheng.A Representation Generation Approach of Transmission Gear Based on Conditional Generative Adversarial Network. Actuators, 2021,10,86: 1-19.

https://doi.org/10.3390/act10050086.

[38]Awan S E , Bennamoun M , Sohel F , et al. Imputation of Missing Data with Class Imbalance using

Conditional Generative Adversarial Networks[J]. Neurocomputing453(2021):164-171.https://doi.org/10.1016/j.n eucom.2021.04.010.

[39]He W, He Y, Li B . Generative Adversarial Networks With Comprehensive Wavelet Feature for Fault Diagnosis of Analog Circuits[J]. IEEE Transactions on Instrumentation and Measurement, 2020, 69(9):6640-6650. 
[40] Rw A , Sz B , Zc C , et al. Enhanced generative adversarial network for extremely imbalanced fault diagnosis of rotating machine[J]. Measurement, 2021.

[41]A. Radford, L. Metz, and S. Chintala. Unsupervised representation learning with deep convolutional generative adversarial networks. arXiv preprint arXiv:1511.06434, 2015. 2, 3, 16

[42] Yu J , Guo Z . Remaining useful life prediction of planet bearings based on conditional deep recurrent generative adversarial network and action discovery[J]. Journal of Mechanical Science and Technology, 2021.

[43]Wu Q , Chen Y , Meng J . DCGAN Based Data Augmentation for Tomato Leaf Disease Identification[J]. IEEE Access, 2020, PP(99):1-1.

[44] Shao S,McAleer S,Yan R,et al.Highly-accurate machine fault diagnosis using deep transfer learning[J].IEEE Transactions on Industrial Informatics, 2019,15(4):2446-2455.

[45]Chen Z Q,Li C,Sanchez R V.Gearbox fault identification and classification with convolutional neural networks[J]. Shock and Vibration,2015,2015.

[46] Bharti V , Biswas B , Shukla K K . EMOCGAN: a novel evolutionary multiobjective cyclic generative adversarial network and its application to unpaired image translation[J]. Neural Computing and Applications, 2021:1-15.

[47]Mao W , Chen J , Chena Y, et al. Construction of Health Indicators for Rotating Machinery Using Deep Transfer Learning With Multiscale Feature Representation[J]. IEEE Transactions on Instrumentation and Measurement, 2021, PP(99):1-1.

[48] Imbalanced Fault Classification of Bearing via Wasserstein Generative Adversarial Networks with Gradient Penalty[J]. Shock and Vibration, 2020, 2020(6):1-14.

[49] Hashemizadehkolowri S K , Chen R R , Adluru G , et al. Simultaneous multi-slice image reconstruction using regularized image domain split slice-GRAPPA for diffusion MRI[J]. Medical Image Analysis, 2021, 70(2):102000.

[50]Shao H , Jiang H , Zhang X , et al. Rolling bearing fault diagnosis using an optimization deep belief network[J]. Measurement Science and Technology, 2015, 26(11):115002.

[51]A K N R , B A Y , C H K, et al. Gearbox Fault Diagnosis based on Multi-Scale Deep Residual Learning and Stacked LSTM Model[J]. Measurement, 2021,186:110099.

[52]Zhao K, Jiang H , Wu Z, et al. A novel transfer learning fault diagnosis method based on Manifold Embedded Distribution Alignment with a little labeled data[J]. Journal of Intelligent Manufacturing, 2020(2):1-15. 